\title{
Resolvent Estimates for High-Contrast Elliptic Problems with Periodic Coefficients
}

\author{
K. D. CHEREDNiCHENKo \& S. COOPER
}

Communicated by F. LIN

\begin{abstract}
We study the asymptotic behaviour of the resolvents $\left(\mathcal{A}^{\varepsilon}+I\right)^{-1}$ of elliptic second-order differential operators $\mathcal{A}^{\varepsilon}$ in $\mathbb{R}^{d}$ with periodic rapidly oscillating coefficients, as the period $\varepsilon$ goes to zero. The class of operators covered by our analysis includes both the "classical" case of uniformly elliptic families (where the ellipticity constant does not depend on $\varepsilon$ ) and the "double-porosity" case of coefficients that take contrasting values of order one and of order $\varepsilon^{2}$ in different parts of the period cell. We provide a construction for the leading order term of the "operator asymptotics" of $\left(\mathcal{A}^{\varepsilon}+I\right)^{-1}$ in the sense of operator-norm convergence and prove order $O(\varepsilon)$ remainder estimates.
\end{abstract}

\section{Introduction}

The subject of the present article is the investigation of analytical properties of partial differential equations (PDE) of a special kind that emerge in the mathematical theory of homogenisation for periodic composites. The study of composite media has been attracting interest since the middle of the last century (see for example $\S 9$ of the monograph [11], where some heuristic relationships for the overall properties of mixtures are discussed), although the question of "averaging" the microstructure in order to get intuitively expected macroscopic quantities goes back a few more decades still. In the early 1970's a number of works appeared concerning the analysis of PDE with periodic rapidly oscillating coefficients, which could be thought of as the simplest, yet already mathematically challenging, object representing the idea of a composite structure. For a classical overview of the related developments we refer the reader to the books $[2,8]$.

In the following years a large amount of literature followed, extending homogenisation theory in various directions. One of the central themes of this activity has been in understanding the relative strength of various notions of convergence in 
terms of characterising the homogenised medium. Unlike in the "classical" case of uniformly elliptic PDE, whose solutions are compact in the usual Sobolev spaces $W^{l, p}$, non-uniformly elliptic problems offer a variety of descriptions for the homogenised medium that depend on the notion of convergence used. From the computational point of view, one is presented with the question of what approaches yield controlled error estimates for the difference between the original and homogenised solutions.

A number of results have been obtained recently concerning the difference, in the operator norm, between the resolvent of the differential operator representing the original heterogeneous medium

$$
-\operatorname{div}\left(A\left(\frac{x}{\varepsilon}\right) \nabla u\right), \quad u \in D^{\varepsilon} \subset L^{2}(\Omega), \quad \varepsilon>0,
$$

and the resolvent of the operator representing the "homogenisation limit"

$$
-\operatorname{div}\left(A^{\text {hom }} \nabla u\right), \quad u \in D^{\text {hom }} \subset L^{2}(\Omega) .
$$

Here $\Omega$ is an open connected subset of $\mathbb{R}^{d}$, the matrix function $A$ is $[0,1)^{d}$ periodic, bounded and uniformly positive definite, the constant matrix $A^{\text {hom }}$ represents the homogenised medium, and $D^{\varepsilon}, D^{\text {hom }}$ denote the domains of the corresponding operators. While a basic order $O(\sqrt{\varepsilon})$ estimate for this setup has been known for a long time, see for example [8], one should in principle expect the better rate of convergence of order $O(\varepsilon)$ suggested by the formal asymptotic analysis (assuming that the domain $\Omega$ is sufficiently regular). The work [3] contains the related result for problems in the whole space $\left(\Omega=\mathbb{R}^{d}\right)$, via a combination of spectral theoretic machinery based on the Bloch fibre decomposition of periodic PDE and asymptotic analysis. Earlier works $[4,13]$ used similar ideas to prove resolvent convergence, but they did not go as far as getting the order $O(\varepsilon)$ operator norm estimates. The more recent papers $[10,16]$ use different techniques to show an improved rate of convergence of order $O\left(\varepsilon|\log \varepsilon|^{\sigma}\right), \sigma>0$, for problems in bounded domains: [16] for scalar equations and [10] for systems. Finally, the papers $[5,12]$ prove the "expected" order $O(\varepsilon)$ convergence for such problems: [5] by using the method of periodic unfolding (and only for scalar problems), and [12] by combining the earlier results of [3] with some elements of the approach of [16] (and including the case of a system).

The focus of the present paper is on obtaining operator-norm resolvent-type estimates for a class of non-uniformly elliptic problems of the "double porosity" type, where the matrix $A=A^{\varepsilon}$ takes values of order one and of order $\varepsilon^{2}$ in mutually complementary parts of the "unit cell" $[0,1)^{d}$. The presence of multiscale effects for such problems was first highlighted in the paper [1]. An analysis of the relation between these effects and the resolvent behaviour of double-porosity problems was carried out in [14].

The earlier results [3] concerning resolvent estimates for (1.1)-(1.2) are based on the analysis of spectral projections of the associated operators in a neighbourhood of zero. This approach does not suffice in the double porosity case as all spectral projections provide a leading-order contribution to the behaviour of the resolvent as $\varepsilon \rightarrow 0$. Bearing this in mind, we analyse the asymptotic behaviour of the operator 
fibres provided by the Bloch decomposition. As was observed by [6], the pointwise limit of the fibres is insufficient for norm-resolvent estimates. We show that in fact the convergence of the individual fibre resolvents is non-uniform with respect to the quasimomentum $\varkappa \in[0,2 \pi)^{d}$. This effect is due to the presence of a "boundary layer" in the neighbourhood of the origin $\varkappa=0$, where the asymptotics for each fixed $\varkappa$ fails to be valid. To obtain uniform estimates in this neighbourhood we study the asymptotics for the "rescaled fibres" parametrised by $\theta=\varkappa / \varepsilon$. The corresponding inner expansion is coupled to the pointwise outer expansion in a matching region where neither expansion is uniform.

We briefly outline the structure of the paper. In Section 2 we introduce the sequence of problems we analyse. In Section 3 we recall the notions of the direct fibre decomposition and of the associated Gelfand transform. Section 4 contains the formulation of our main result using these notions. In Section 5 we describe the resolvent asymptotics in the "inner" region for relevant values of the quasimomentum $\theta \in \varepsilon^{-1}[0,2 \pi)^{d}$. In Section 6 we introduce spaces $V(\varkappa) \subset H_{\#}^{1}(Q)$, $\varkappa \in[0,2 \pi)^{d}$, which play a key role in our construction. We also prove some lemmas used in the proof of the main result, namely a special Poincaré-type inequality for the projection on the space orthogonal to $V(\varkappa)$ with respect to the inner product of $H_{\#}^{1}(Q)$, as well as several elliptic estimates that are uniform in $\theta$. Section 7 is devoted to the proof of our main result (Theorem 4.1), which consists of two pieces of analysis, in the inner region $|\theta| \leq 1$ and in its complement $|\theta|>1$. In Section 8 we discuss the "outer" region $|\theta| \geq \varepsilon^{-1 / 2}$ and show that the inner and outer approximations jointly are only sufficient to obtain a norm-resolvent estimate of order $O\left(\varepsilon^{\alpha}\right), \alpha \in(0,1)$. In Section 9 we calculate the limit of the spectra of the operators $-\operatorname{div}\left(A^{\varepsilon}(\cdot / \varepsilon) \nabla\right)$ and explain its relation to an earlier study of [14]. Finally, in Section 10 we show that our main theorem contains as a particular case a result of [3], followed by a discussion of some key points of the work [14] and the relation of its result to our convergence statement.

\section{Problem Setup}

In what follows we study the problem

$$
-\operatorname{div}\left(A^{\varepsilon}\left(\frac{x}{\varepsilon}\right) \nabla u\right)+u=f, \quad f \in L^{2}\left(\mathbb{R}^{d}\right) .
$$

In the above equation

$$
A^{\varepsilon}=A_{1}+\varepsilon^{2} A_{0}
$$

where $A_{0}, A_{1}$ are $Q$-periodic symmetric $(d \times d)$-matrix functions with entries in $L^{\infty}(Q)$. We assume that $A_{0} \geq v I, v>0$ and that $A_{1} \geq v I$ on a Lipschitz open set $Q_{1} \subset Q:=[0,1)^{d}$ (the "stiff" component of the composite) with $A_{1}=0$ on the interior of $Q \backslash Q_{1}$ (the "soft" component), which we denote by $Q_{0}$. We also assume that $\bar{Q}_{0} \subset(0,1)^{d}$, which implies, in particular, that the set $\cup_{n \in \mathbb{Z}^{d}}\left(Q_{1}+n\right)$ is connected in $\mathbb{R}^{d}$. 
We next recall the construction of the operator $\mathcal{A}^{\varepsilon}$ associated with (2.1). The closed sesquilinear form

$$
\mathfrak{a}^{\varepsilon}(u, v)=\int_{\mathbb{R}^{d}} A^{\varepsilon}\left(\frac{x}{\varepsilon}\right) \nabla u(x) \cdot \overline{\nabla v(x)} \mathrm{d} x, \quad u, v \in H^{1}\left(\mathbb{R}^{d}\right),
$$

is symmetric and non-negative in $L^{2}\left(\mathbb{R}^{d}\right)$, hence it generates a self-adjoint operator $\mathcal{A}^{\varepsilon}$ whose domain $D\left(\mathcal{A}^{\varepsilon}\right)$ is dense in $L^{2}\left(\mathbb{R}^{d}\right)$ and whose action is described by the identity $\left(\mathcal{A}^{\varepsilon} u, v\right)_{L^{2}\left(\mathbb{R}^{d}\right)}=\mathfrak{a}^{\varepsilon}(u, v)$ for $u \in D\left(\mathcal{A}^{\varepsilon}\right), v \in H^{1}\left(\mathbb{R}^{d}\right)$. The solution $u=u^{\varepsilon}$ to (2.1) is understood as the result of applying the resolvent of $\mathcal{A}^{\varepsilon}$ to $f$, that is $u_{\varepsilon}=\left(\mathcal{A}^{\varepsilon}+I\right)^{-1} f$. The last formula is well defined for any $f \in L^{2}\left(\mathbb{R}^{d}\right)$ : indeed, the operator $\mathcal{A}^{\varepsilon}+I$ is clearly bounded below by $I$, hence it is injective, and the only element $g \in L^{2}\left(\mathbb{R}^{d}\right)$ orthogonal to the image of $\mathcal{A}^{\varepsilon}+I$ is $g=0$ by virtue of the fact that the form $\mathfrak{a}^{\varepsilon}(u, v)+(u, v)_{L^{2}\left(\mathbb{R}^{d}\right)}, u, v \in H^{1}\left(\mathbb{R}^{d}\right)$, is positive. The same fact implies that the resolvent $\left(\mathcal{A}^{\varepsilon}+I\right)^{-1}$ is a bounded operator.

Throughout the text we denote by $H_{\#}^{1}(Q)$ the space of $H_{\text {loc }}^{1}\left(\mathbb{R}^{d}\right)$ functions that are $Q$-periodic and by $H_{\#}^{-1}(Q)$ its dual. For a normed space $X$ and its dual $X^{*}$, we write $\langle f, v\rangle$ for the action of $f \in X^{*}$ on $v \in X$. We use the letter $C$ for any positive constant whose exact value may vary from line to line.

\section{Bloch Formulation and Gelfand Transform}

Using a procedure similar to the above definition of $\left(\mathcal{A}^{\varepsilon}+I\right)^{-1}$, for each $\theta \in \varepsilon^{-1} Q^{\prime}$, where $Q^{\prime}:=[0,2 \pi)^{d}$, we define $u_{\theta}^{\varepsilon} \in H_{\#}^{1}(Q)$ as the solution to

$$
-\varepsilon^{-2}(\nabla+\mathrm{i} \varepsilon \theta) \cdot A^{\varepsilon}(\nabla+\mathrm{i} \varepsilon \theta) u_{\theta}^{\varepsilon}+u_{\theta}^{\varepsilon}=F, \quad F \in L^{2}(Q) .
$$

In other words, for all $\theta \in \varepsilon^{-1} Q^{\prime}$ one has $u_{\theta}^{\varepsilon}=\left(\mathcal{B}_{\varepsilon, \theta}+I\right)^{-1} F$, where the operators $\mathcal{B}_{\varepsilon, \theta}$ are generated by the closed sesquilinear forms

$$
\mathfrak{b}_{\varepsilon, \theta}(u, v)=\int_{Q}\left(\varepsilon^{-2} A_{1}+A_{0}\right)(\nabla+\mathrm{i} \varepsilon \theta) u \cdot \overline{(\nabla+\mathrm{i} \varepsilon \theta) v}, \quad u, v \in H_{\#}^{1}(Q) .
$$

Lemma 3.1. For each $\varepsilon>0$ there is a unitary map $\mathcal{U}_{\varepsilon}: L^{2}\left(\mathbb{R}^{d}\right) \rightarrow L^{2}\left(\varepsilon^{-1} Q^{\prime} \times Q\right)$ such that

$$
\mathcal{U}_{\varepsilon}\left(\mathcal{A}^{\varepsilon}+I\right)^{-1} \mathcal{U}_{\varepsilon}^{-1}=\int_{\varepsilon^{-1} Q^{\prime}}^{\oplus}\left(\mathcal{B}_{\varepsilon, \theta}+I\right)^{-1} \mathrm{~d} \theta
$$

that is for all $f \in L^{2}\left(\mathbb{R}^{d}\right)$ the formula $\left(\mathcal{A}^{\varepsilon}+I\right)^{-1} f=\mathcal{U}_{\varepsilon}^{-1} g$ holds, where for each $\theta \in \varepsilon^{-1} Q^{\prime}$ one has $g(\theta, \cdot)=\left(\mathcal{B}_{\varepsilon, \theta}+I\right)^{-1}\left(\mathcal{U}_{\varepsilon} f\right)(\theta, \cdot)$.

Proof. For a given $\varepsilon>0$ set

$$
\left(\mathcal{U}_{\varepsilon} f\right)(\theta, y):=\left(\frac{\varepsilon^{2}}{2 \pi}\right)^{d / 2} \sum_{n \in \mathbb{Z}^{d}} f(\varepsilon(y+n)) \mathrm{e}^{-\mathrm{i} \varepsilon \theta \cdot(y+n)}, \quad \theta \in \varepsilon^{-1} Q^{\prime}, \quad y \in Q .
$$


Note that for each $\varepsilon$ the operator $\mathcal{U}_{\varepsilon}$ is the composition $\mathcal{T}_{\varepsilon} \mathcal{G}_{\varepsilon}$ of a scaled version of the usual Gelfand transform $\mathcal{G}_{\varepsilon}: L^{2}\left(\mathbb{R}^{d}\right) \rightarrow L^{2}\left(\varepsilon^{-1} Q^{\prime} \times \varepsilon Q\right)$, given by

$$
\left(\mathcal{G}_{\varepsilon} f\right)(\theta, z):=\left(\frac{\varepsilon}{2 \pi}\right)^{d / 2} \sum_{n \in \mathbb{Z}^{d}} f(z+\varepsilon n) \mathrm{e}^{-\mathrm{i} \theta \cdot(z+\varepsilon n)}, \quad \theta \in \varepsilon^{-1} Q^{\prime}, z \in \varepsilon Q,
$$

and the scaling transform $\mathcal{T}_{\varepsilon}: L^{2}\left(\varepsilon^{-1} Q^{\prime} \times \varepsilon Q\right) \rightarrow L^{2}\left(\varepsilon^{-1} Q^{\prime} \times Q\right)$ given by

$$
\left(\mathcal{T}_{\varepsilon} h\right)(\theta, y):=\varepsilon^{d / 2} h(\theta, \varepsilon y) .
$$

The inverse $\mathcal{U}_{\varepsilon}^{-1}$ is the composition $\mathcal{G}_{\varepsilon}^{-1} \mathcal{T}_{\varepsilon}^{-1}$ of the inverse of $\mathcal{T}_{\varepsilon}$ given by

$$
\left(\mathcal{T}_{\varepsilon}^{-1} g\right)(\theta, x):=\varepsilon^{-d / 2} g(\theta, x / \varepsilon), \quad \theta \in \varepsilon^{-1} Q^{\prime}, x \in \mathbb{R}^{d},
$$

and the inverse of $\mathcal{G}_{\varepsilon}$ given by

$$
\left(\mathcal{G}_{\varepsilon}^{-1} h\right)(x)=\left(\frac{\varepsilon}{2 \pi}\right)^{d / 2} \sum \int_{\varepsilon^{-1} Q^{\prime}} h(\theta, x) \mathrm{e}^{\mathrm{i} \theta \cdot x} \mathrm{~d} \theta, \quad x \in \mathbb{R}^{d} .
$$

The map $\mathcal{U}_{\varepsilon}$ is unitary since the corresponding property clearly holds for $\mathcal{T}_{\varepsilon}$ and is well known for $\mathcal{G}_{\varepsilon}$, see for example [2].

\section{Homogenised Operator in $\theta$-Representation and the Main Convergence Result}

First, we introduce a $\theta$-parametrised operator family that plays a central role in our analysis of the operators $\mathcal{A}^{\varepsilon}$ as $\varepsilon \rightarrow 0$.

We denote $\mathcal{H}_{0}:=\mathbb{C} \times H_{0}^{1}\left(Q_{0}\right)$, and for each $\varepsilon>0$ and $\theta \in \varepsilon^{-1} Q^{\prime}$ consider the sesquilinear form

$$
\begin{aligned}
\mathfrak{b}_{\varepsilon, \theta}^{\text {hom }}((c, u),(d, v)):= & A{ }^{\text {hom }_{\theta} \cdot \theta c \bar{d}} \\
& +\int_{Q} A_{0}(\nabla+\mathrm{i} \varepsilon \theta) u \cdot \overline{(\nabla+\mathrm{i} \varepsilon \theta) v}, \quad(c, u),(d, v) \in \mathcal{H}_{0},
\end{aligned}
$$

where $A^{\text {hom }}$ is the usual homogenised matrix

$$
A^{\text {hom }} \xi \cdot \xi=\min _{u \in H_{\#}^{1}(Q)} \int_{Q} A_{1}(\xi+\nabla u) \cdot \overline{(\xi+\nabla u)}, \quad \xi \in \mathbb{R}^{d} .
$$

Note that the matrix $A^{\text {hom }}$ is positive definite. Indeed, using the ellipticity assumption on $A_{1}$ one has, for $\xi \in \mathbb{R}^{d}$,

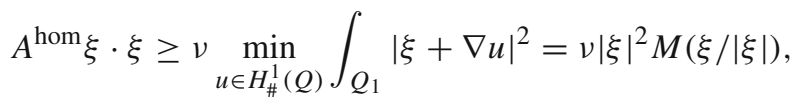

where the function

$$
M(\eta):=\min _{u \in H_{\#}^{1}(Q)} \int_{Q_{1}}|\eta+\nabla u|^{2}, \quad|\eta|=1
$$

has a positive minimum $M_{\min }$, hence $A^{\text {hom }} \geq v M_{\min }$. 
In what follows we also denote

$$
\mathcal{L}:=\left\{c+\widetilde{u}: c \in \mathbb{C}, \widetilde{u} \in L^{2}(Q),\left.\widetilde{u}\right|_{Q_{1}}=0\right\} \subset L^{2}(Q),
$$

and use the invertible "identification" map $\mathcal{I}: \mathbb{C} \times L^{2}\left(Q_{0}\right) \rightarrow \mathcal{L}$ that takes each pair $(c, u)$ to the function $c+\widetilde{u} \in \mathcal{L}$ with $\widetilde{u}=u$ on $Q_{0}$ and $\widetilde{u}=0$ on $Q_{1}$.

We next define operators $\mathcal{B}_{\varepsilon, \theta}^{\text {hom }}$ in the Hilbert space $\mathbb{C} \times L^{2}\left(Q_{0}\right)$ equipped with the inner product $((c, u),(d, v))_{0}=(\mathcal{I}(c, u), \mathcal{I}(d, v))_{L^{2}(Q)}$. These operators are associated, for each value of $\theta \in \varepsilon^{-1} Q^{\prime}$, with the forms $\mathfrak{b}_{\varepsilon, \theta}^{\text {hom }}$ by means of the identity

$$
\left(\mathcal{B}_{\varepsilon, \theta}^{\mathrm{hom}}(c, u),(d, v)\right)_{0}=\mathfrak{b}_{\varepsilon, \theta}^{\mathrm{hom}}((c, u),(d, v)), \quad(d, v) \in \mathcal{H}_{0},
$$

where the pairs $(c, u)$ are taken from the maximal possible domain $D\left(\mathcal{B}_{\varepsilon, \theta}^{\text {hom }}\right)$, which can be shown to be dense in $\mathcal{H}_{0}$ and hence in $\mathbb{C} \times L^{2}\left(Q_{0}\right)$.

The operators $\mathcal{B}_{0, \theta}^{\text {hom }}$ can be viewed, roughly speaking, as the $\theta$-components of the Fourier transform of the two-scale homogenised operator, see Section 10 below, with respect to the "macroscopic" variable. However, as we also discuss in the same section, in order to obtain operator-norm resolvent estimates it is important to deal with a suitable "truncation" of this Fourier transform that restricts the Fourier variable $\theta$ to the set $\varepsilon^{-1} Q^{\prime}$. From this perspective the analysis below can be viewed as a rigorous procedure for such a truncation. Note that in view of the non-uniform behaviour of these truncations as $\varepsilon \rightarrow 0$, as we discuss in Sections 1 and 8 , the expression $\varepsilon \theta$ in (4.1) can not be set to zero in the region $|\theta| \geq 1$, hence the dependence of the operators $\mathcal{B}_{\varepsilon, \theta}^{\text {hom }}$ on $\varepsilon$.

We also denote by $\mathcal{P}$ the orthogonal projection of the Hilbert space $L^{2}\left(\varepsilon^{-1} Q^{\prime} \times Q\right)$ onto its closed subspace

$$
\begin{aligned}
& \left\{c+g: c \in L^{2}\left(\varepsilon^{-1} Q^{\prime}\right), g \in L^{2}\left(\varepsilon^{-1} Q^{\prime} \times Q\right),\right. \\
& \left.\quad g(\theta, y)=0 \text { for almost all }(\theta, y) \in \varepsilon^{-1} Q^{\prime} \times Q_{1}\right\},
\end{aligned}
$$

and by $\mathcal{P}_{\mathrm{f}}$ its analogue on each "fibre", the orthogonal projection of $L^{2}(Q)$ onto $\mathcal{L}$.

The main result of the present paper is as follows.

Theorem 4.1. The resolvents of the operator family $\mathcal{A}^{\varepsilon}$ are asymptotically close as $\varepsilon \rightarrow 0$ to the family

$$
\mathcal{R}^{\varepsilon}:=\mathcal{U}_{\varepsilon}^{-1} \int_{\varepsilon^{-1} Q^{\prime}}^{\oplus} \mathcal{I}\left(\mathcal{B}_{\varepsilon, \theta}^{\mathrm{hom}}+I\right)^{-1} \mathcal{I}^{-1} \mathrm{~d} \theta \mathcal{P} \mathcal{U}_{\varepsilon},
$$

where the corresponding approximation error is of order $O(\varepsilon)$. More precisely, there exists a constant $C>0$, independent of $\varepsilon$, such that

$$
\left\|\left(\mathcal{A}^{\varepsilon}+I\right)^{-1}-\mathcal{R}^{\varepsilon}\right\|_{L^{2}\left(\mathbb{R}^{d}\right) \rightarrow L^{2}\left(\mathbb{R}^{d}\right)} \leq C \varepsilon .
$$

Note that the operator $\mathcal{R}^{\varepsilon}$ can also be written as

$$
\mathcal{R}^{\varepsilon}=\mathcal{U}_{\varepsilon}^{-1} \int_{\varepsilon^{-1} Q^{\prime}}^{\oplus} \mathcal{I}\left(\mathcal{B}_{\varepsilon, \theta}^{\mathrm{hom}}+I\right)^{-1} \mathcal{I}^{-1} \mathcal{P}_{\mathrm{f}} \mathrm{d} \theta \mathcal{U}_{\varepsilon},
$$

which follows from the definitions of the projection operators $\mathcal{P}$ and $\mathcal{P}_{\mathrm{f}}$. 


\section{The Inner Expansion and Principal Term for $\mathcal{B}_{\varepsilon, \theta}^{\text {hom }}$ in the Inner Region $|\theta| \leq 1$}

In this section we provide an explicit representation for the behaviour in $\varepsilon$ of the operators $\mathcal{B}_{\varepsilon, \theta}^{\text {hom }}$ in the region $|\theta| \leq 1$. We refer to this expansion as the inner expansion and to its region of validity as the inner region.

Let us consider an asymptotic expansion for solutions to (3.1) of the form

$$
u_{\theta}^{\varepsilon}=\sum_{n=0}^{\infty} \varepsilon^{n} u_{\theta}^{(n)}, \quad u_{\theta}^{(n)} \in H_{\#}^{1}(Q), \quad n=0,1,2, \ldots
$$

Substituting (5.1) into (3.1) and comparing the coefficients in front of $\varepsilon^{-2}$ on both sides of the resulting equation we find

$$
\nabla \cdot A_{1} \nabla u_{\theta}^{(0)}=0
$$

or, equivalently,

$$
u_{\theta}^{(0)} \in V:=\left\{u \in H_{\#}^{1}(Q) \quad \mid A_{1} \nabla u=0\right\},
$$

which is a space naturally isometric to $\mathcal{H}_{0}$ via the mapping $\mathcal{I}$ defined above:

$$
\mathcal{H}_{0} \ni(c, v) \mapsto u=c+\widetilde{v} \in V
$$

where, as before, $\widetilde{v}=v$ on $Q_{0}$ and $\widetilde{v}=0$ on $Q_{1}$. This implies that $u_{\theta}^{(0)}=c_{\theta}^{(0)}+v_{\theta}^{(0)}$, where the pair $\left(c_{\theta}^{(0)}, v_{\theta}^{(0)}\right)$ belongs to $\mathcal{H}_{0}$.

Further, comparing the coefficients in front of $\varepsilon^{-1}$ and using (5.3) yields

$$
-\nabla \cdot A_{1} \nabla u_{\theta}^{(1)}=\mathrm{i} \nabla \cdot A_{1} \theta c_{\theta}^{(0)} .
$$

Introducing "unit-cell solutions" $N_{k}, k=1, \ldots, d$, that satisfy

$$
-\sum_{i, j=1}^{d} \partial_{i}\left(\left(A_{1}\right)_{i j} \partial_{j} N_{k}\right)=\sum_{j=1}^{d} \partial_{j}\left(A_{1}\right)_{k j},
$$

we note that, up to an arbitrary additive constant, one has

$$
u_{\theta}^{(1)}=\mathrm{i} \sum_{j=1}^{d} N_{j} \theta_{j} c_{\theta}^{(0)} .
$$

The concrete choice of the constant added to the right-hand side of (5.6) plays an important role in the justification of the asymptotic expansion, which we discuss in Section 6.2 (see proof of Lemma 6.3).

Finally, comparing the coefficients in front of $\varepsilon^{0}$ yields an equation for $u_{\theta}^{(2)}$ as follows

$$
-\nabla \cdot A_{1} \nabla u_{\theta}^{(2)}=F_{\theta}
$$


where

$$
F_{\theta}:=F+\mathrm{i}\left(\nabla \cdot A_{1} \theta+\theta \cdot A_{1} \nabla\right) u_{\theta}^{(1)}+\nabla \cdot A_{0} \nabla u_{\theta}^{(0)}-\theta \cdot A_{1} \theta c_{\theta}^{(0)}-u_{\theta}^{(0)} .
$$

Solvability of (5.7) requires that $\left\langle F_{\theta}, v\right\rangle=0$ for all $v \in V$. The formula (5.6) and the solvability condition for (5.7) imply that $u_{\theta}^{(0)}=c_{\theta}^{(0)}+v_{\theta}^{(0)}$, where the pair $\left(c_{\theta}^{(0)}, v_{\theta}^{(0)}\right) \in \mathcal{H}_{0}$ satisfies the identity

$$
\begin{aligned}
& A^{\text {hom }} \theta \cdot \theta c_{\theta}^{(0)} \bar{d}+\int_{Q} A_{0} \nabla v_{\theta}^{(0)} \cdot \overline{\nabla \varphi}+\int_{Q}\left(c_{\theta}^{(0)}+v_{\theta}^{(0)}\right) \overline{(d+\varphi)} \\
& =\int_{Q} \mathcal{P}_{\mathrm{f}} F \overline{(d+\varphi)} \quad \forall(d, \varphi) \in \mathcal{H}_{0} .
\end{aligned}
$$

Following the method outlined in Section 4 for the construction of $\mathcal{B}_{\varepsilon, \theta}^{\text {hom }}$, we introduce the operator $\mathcal{B}_{0, \theta}^{\text {hom }}$ associated with the problem (5.9) such that $\left(c_{\theta}^{(0)}, v_{\theta}^{(0)}\right)=$ $\left(\mathcal{B}_{0, \theta}^{\text {hom }}+I\right)^{-1} \mathcal{I}^{-1} \mathcal{P}_{\mathrm{f}} F$. The next result shows that $\mathcal{B}_{0, \theta}^{\text {hom }}$ is $\varepsilon$-close in norm to $\mathcal{B}_{\varepsilon, \theta}^{\text {hom }}$ in the inner region of $\theta$.

Lemma 5.1. There exists $C>0$ such that the estimate

$$
\left\|\mathcal{I}\left(\mathcal{B}_{0, \theta}^{\mathrm{hom}}+I\right)^{-1} \mathcal{I}^{-1} \mathcal{P}_{\mathrm{f}}-\mathcal{I}\left(\mathcal{B}_{\varepsilon, \theta}^{\mathrm{hom}}+I\right)^{-1} \mathcal{I}^{-1} \mathcal{P}_{\mathrm{f}}\right\|_{L^{2}(Q) \rightarrow L^{2}(Q)} \leq C \varepsilon
$$

holds for all $\theta \in \varepsilon^{-1} Q^{\prime}$ satisfying the inequality $|\theta| \leq 1$.

Proof. For each $\theta$ as in the lemma, consider the pairs $(c, v)=\left(\mathcal{B}_{0, \theta}^{\text {hom }}+I\right)^{-1} \mathcal{I}^{-1} P_{\mathrm{f}} F$ and $\left(c_{\varepsilon}, v_{\varepsilon}\right)=\left(\mathcal{B}_{\varepsilon, \theta}^{\text {hom }}+I\right)^{-1} \mathcal{I}^{-1} P_{\mathrm{f}} F$, that is

$$
\begin{aligned}
& A^{\mathrm{hom}} \theta \cdot \theta c \bar{d}+\int_{Q} A_{0} \nabla v \cdot \overline{\nabla \varphi}+\int_{Q}(c+v) \overline{(d+\varphi)} \\
& =\int_{Q} \mathcal{P}_{\mathrm{f}} \overline{F(d+\varphi)} \quad \forall(d, \varphi) \in \mathcal{H}_{0},
\end{aligned}
$$

and

$$
\begin{aligned}
& A^{\mathrm{hom}} \theta \cdot \theta c_{\varepsilon} \bar{d}+\int_{Q} A_{0}(\nabla+\mathrm{i} \varepsilon \theta) v_{\varepsilon} \cdot \overline{(\nabla+\mathrm{i} \varepsilon \theta) \varphi}+\int_{Q}\left(c_{\varepsilon}+v_{\varepsilon}\right) \overline{(d+\varphi)} \\
& \quad=\int_{Q} \mathcal{P}_{\mathrm{f}} \overline{F(d+\varphi)} \quad \forall(d, \varphi) \in \mathcal{H}_{0}
\end{aligned}
$$

By setting $(d, \varphi)=\left(c_{\varepsilon}, v_{\varepsilon}\right)$ in (5.11) and noting that $v_{\varepsilon} \in H_{0}^{1}\left(Q_{0}\right)$ we arrive at the a priori bound

$$
\left\|\nabla v_{\varepsilon}\right\|_{L^{2}\left(Q_{0}\right)} \leq C\|F\|_{L^{2}(Q)}
$$

for some constant $C$. 
To prove the result we show that for $u_{\varepsilon}:=\mathcal{I}\left(c_{\varepsilon}, v_{\varepsilon}\right)$ and $u:=\mathcal{I}(c, v)$ there exists a constant $C>0$ independent of $\varepsilon, \theta$ such that

$$
\left\|u_{\varepsilon}-u\right\|_{H^{1}(Q)} \leq C \varepsilon|\theta|\|F\|_{L^{2}(Q)} .
$$

Subtracting (5.10) from (5.11) implies

$$
\begin{aligned}
& A^{\mathrm{hom}} \theta \cdot \theta\left(c_{\varepsilon}-c\right) \bar{d}+\int_{Q} A_{0} \nabla\left(v_{\varepsilon}-v\right) \cdot \overline{\nabla \varphi}+\int_{Q}\left(c_{\varepsilon}+v_{\varepsilon}-c-v\right) \overline{(d+\varphi)} \\
& =-\int_{Q} A_{0} \nabla v_{\varepsilon} \cdot \overline{\mathrm{i} \varepsilon \theta \varphi}-\int_{Q} A_{0} \mathrm{i} \varepsilon \theta v_{\varepsilon} \cdot \overline{(\nabla+\mathrm{i} \varepsilon \theta) \varphi} \quad \forall(d, \varphi) \in \mathcal{H}_{0} .
\end{aligned}
$$

Setting $(d, \varphi)=\left(c_{\varepsilon}-c, 0\right)$ in $(5.14)$ gives

$$
\left(A^{\mathrm{hom}} \theta \cdot \theta+1\right)\left(c_{\varepsilon}-c\right) \overline{\left(c_{\varepsilon}-c\right)}=-\left(\overline{c_{\varepsilon}-c}\right) \int_{Q}\left(v_{\varepsilon}-v\right)
$$

hence

$$
\left|c_{\varepsilon}-c\right| \leq C\left\|\nabla\left(v_{\varepsilon}-v\right)\right\|_{L^{2}\left(Q_{0}\right)},
$$

since $v_{\varepsilon}, v \in H_{0}^{1}\left(Q_{0}\right)$.

Setting $(d, \varphi)=\left(c_{\varepsilon}-c, v_{\varepsilon}-v\right)$ in (5.14) gives

$$
\begin{aligned}
\left\|\nabla\left(v_{\varepsilon}-v\right)\right\|_{L^{2}\left(Q_{0}\right)}^{2} \leq & C \int_{Q} A_{0} \nabla\left(v_{\varepsilon}-v\right) \cdot \nabla\left(v_{\varepsilon}-v\right) \\
\leq & C\left[-\int_{Q} A_{0} \nabla v_{\varepsilon} \cdot \overline{\mathrm{i} \varepsilon \theta\left(v_{\varepsilon}-v\right)}\right. \\
& \left.\quad-\int_{Q} A_{0} \mathrm{i} \varepsilon \theta v_{\varepsilon} \cdot \overline{(\nabla+\mathrm{i} \varepsilon \theta)\left(v_{\varepsilon}-v\right)}\right] \\
\leq & C \varepsilon|\theta|\left\|\nabla v_{\varepsilon}\right\|_{L^{2}\left(Q_{0}\right)}\left\|\nabla\left(v_{\varepsilon}-v\right)\right\|_{L^{2}(Q)} .
\end{aligned}
$$

Taking into account (5.12) this implies (5.13), since

$$
\left\|u_{\varepsilon}-u\right\|_{L^{2}(Q)} \leq\left|c_{\varepsilon}-c\right|+\left\|v_{\varepsilon}-v\right\|_{L^{2}\left(Q_{0}\right)} \leq C\left\|\nabla\left(v_{\varepsilon}-v\right)\right\|_{L^{2}\left(Q_{0}\right)} .
$$

\section{Auxiliary Material}

\subsection{Cell Problems}

One of the key elements in the proof of our main result is the analysis of the properties of the following family of auxiliary "cell problems":

$$
-\nabla \cdot A_{1} \nabla w=G, \quad G \in H_{\varkappa}^{-1}(Q):=\left(H_{\varkappa}^{1}(Q)\right)^{*} .
$$


Here $H_{\varkappa}^{1}(Q), \varkappa \in Q^{\prime}$, is the space of $\varkappa$-quasiperiodic functions belonging to $H^{1}(Q)$, that is $u \in H_{\varkappa}^{1}(Q)$ if and only if $u(y)=\exp (\mathrm{i} \varkappa \cdot y) v(y), y \in Q$, where ${ }^{1}$ $v \in H_{\#}^{1}(Q)$. Note that (5.2), (5.4), (5.7) all have the form (6.1) for $\varkappa=0$ with $G=0, G=\mathrm{i} \nabla \cdot A_{1} \theta c_{\theta}^{(0)}, G=F_{\theta}$, respectively.

For a given matrix function $A_{1}$ we consider the space

$$
V(\varkappa):=\left\{v \in H_{\varkappa}^{1}(Q) \mid A_{1} \nabla v=0\right\} .
$$

Note that, for $A_{1}$ satisfying the assumptions prescribed in Section 2, we find

$$
V(\varkappa)= \begin{cases}V & \text { for } \varkappa=0, \\ H_{0}^{1}\left(Q_{0}\right) & \text { for } \varkappa \neq 0 .\end{cases}
$$

A criterion for the existence of solutions to (6.1) is given below by a variant of the Lax-Milgram lemma. The related ideas are inspired by the work [10], where Poncaré-type inequalities similar to (6.2), for the case $\varkappa=0$, were shown to be sufficient for homogenisation (in the strong two-scale resolvent sense) of "partially degenerate problems" with periodic rapidly oscillating coefficients. For the normresolvent asymptotics, however, a $\varkappa$ - dependent version of the inequality is required, which we prove by following the line of the argument of [10].

Lemma 6.1. For all $\varkappa \in Q^{\prime}$, denote by $V(\varkappa)^{\perp}$ the orthogonal complement of $V(\varkappa)$ in $H_{\varkappa}^{1}(Q)$. Then, for all values of $\varkappa$ :

(i) There exists a constant $C>0$ independent of $\varkappa$ such that

$$
\left\|P_{V(\varkappa)^{\perp}} w\right\|_{H^{1}(Q)} \leq C d(\varkappa)\left\|A_{1} \nabla w\right\|_{L^{2}(Q)} \forall w \in H_{\varkappa}^{1}(Q),
$$

where

$$
d(\varkappa)= \begin{cases}1 & \text { for } \varkappa=0 \\ |\varkappa|^{-1} & \text { for } \varkappa \neq 0\end{cases}
$$

and $P_{V(\varkappa)^{\perp}}$ is the orthogonal projection of $H_{\varkappa}^{1}(Q)$ onto $V(\varkappa)^{\perp}$.

(ii) There exists a solution $w \in H_{\varkappa}^{1}(Q)$ to (6.1) if and only if $\langle G, \varphi\rangle=0$ for all $\varphi \in V(\varkappa)$.

(iii) Any solution to (6.1) is unique up to the addition of an element from $V(\varkappa)$ : if $w$ satisfies (6.1) then $w+v$ satisfies (6.1) for any $v \in V(\varkappa)$, and if $w_{1}, w_{2}$ satisfy (6.1) then $w_{1}-w_{2} \in V(\varkappa)$. In particular, if $w$ is a solution to (6.1) then $P_{V(\varkappa)^{\perp}} w$ is the unique part in $V(\varkappa)^{\perp}$ of any solution to (6.1).

Proof. (i) The inequality (6.3) holds if there exists a constant $C>0$ such that for all $u \in H_{\varkappa}^{1}(Q)$ there exists $v \in V(\varkappa)$ such that

$$
\|u-v\|_{H^{1}(Q)} \leq C d(\varkappa) \int_{Q_{1}}|\nabla u|^{2} \mathrm{~d} y .
$$

We shall now verify this for two distinct cases.

${ }^{1} H_{\varkappa}^{1}(Q)$ coincides with $H_{\#}^{1}(Q)$ when $\varkappa=0$. 
Case 1: $\varkappa=0$. For fixed $u \in H_{\#}^{1}(Q)$, denote $\widetilde{u} \in H_{\#}^{1}(Q)$ to be an extension of $u$ such that

$$
\|\nabla \widetilde{u}\|_{L^{2}(Q)} \leq C\|\nabla u\|_{L^{2}\left(Q_{1}\right)} .
$$

Notice that such an extension exists for connected $Q_{1}$ (cf. [8, Section 3.1]). Defining $v:=u-\tilde{u}+\left|Q_{1}\right|^{-1} \int_{Q_{1}} \tilde{u}$, we see that $v \in V$ and

$$
\|u-v\|_{H^{1}(Q)}=\left\|\widetilde{u}-\frac{1}{\left|Q_{1}\right|} \int_{Q_{1}} \widetilde{u}\right\|_{H^{1}(Q)} \leq C \int_{Q}|\nabla \widetilde{u}|^{2} \leq C \int_{Q_{1}}|\nabla u|^{2},
$$

where the first inequality is a variant of the standard Poincare inequality.

Case 2: $\varkappa \neq 0$. For fixed $u \in H_{\varkappa}^{1}(Q)$, we show there exists $v \in V(\varkappa)$ such that

$$
\|u-v\|_{H^{1}(Q)}^{2} \leq \frac{C}{|\varkappa|^{2}}\|\nabla u\|_{L^{2}\left(Q_{1}\right)}^{2} .
$$

Denoting the map ${ }^{\sim}$ as above, we find that $u-\widetilde{u}=: v \in H_{0}^{1}\left(Q_{0}\right)(=V(\varkappa))$ and

$$
\|u-v\|_{H^{1}(Q)}^{2}=\|\widetilde{u}\|_{H^{1}(Q)}^{2} \leq \frac{C}{|\varkappa|^{2}}\|\nabla \widetilde{u}\|_{L^{2}(Q)}^{2} \leq \frac{C}{|\varkappa|^{2}}\|\nabla u\|_{L^{2}\left(Q_{1}\right)}^{2},
$$

which proves the result. Here we have used the Poincaré-type inequality

$$
\|u\|_{L^{2}(Q)}^{2} \leq \frac{C}{|\varkappa|^{2}}\|\nabla u\|_{L^{2}(Q)}^{2} \quad \forall u \in H_{\varkappa}^{1}(Q),
$$

which is true since $|\varkappa|^{2}$ is the first eigenvalue of the Laplace operator with $\varkappa$-quasiperiodic boundary conditions.

(ii) Let $w$ be a solution of (6.1) and let $\varphi \in V(\varkappa)$. Then, using the symmetry of $A_{1}$ and (6.2),

$$
\langle G, \varphi\rangle=\int_{Q} A_{1} \nabla w \cdot \nabla \varphi=\int_{Q} \nabla w \cdot A_{1} \nabla \varphi=0,
$$

which yields $\langle G, \varphi\rangle=0$ for all $\varphi \in V(\varkappa)$. Conversely, suppose $\langle G, \varphi\rangle=0$ for all $\varphi \in V(\varkappa)$, and seek $w \in H_{\varkappa}^{1}(Q)$ that satisfies (6.1). By (6.4), the identity

$$
\int_{Q} A_{1} \nabla w \cdot \nabla \varphi=\langle G, \varphi\rangle
$$

holds automatically for all $\varphi \in V(\varkappa)$, therefore it is sufficient to verify it for all $\varphi \in V(\varkappa)^{\perp}$. Seeking $w$ in $V(\varkappa)^{\perp}$ reduces the problem to showing that, in the Hilbert space $H:=V(\varkappa)^{\perp}$ with the norm inherited from $H^{1}(Q)$, the problem (6.5) satisfies the conditions of the Lax-Milgram lemma (see example [8, Section 1.1]). As the bilinear form

$$
B[v, w]:=\int_{Q} A_{1} \nabla v \cdot \nabla w
$$

is clearly bounded in $H$, that is for some $C>0$ one has $|B[v, w]| \leq$ $C\|v\|_{H^{1}(Q)}\|w\|_{H^{1}(Q)}$, in order to satisfy the conditions of the Lax-Milgram 
lemma it remains to show that the form $B$ is coercive, that is for some $v>0$ the bound $B[v, v] \geq v\|v\|_{H^{1}(Q)}^{2}$ holds. To this end, note that the boundedness of $A_{1}$ and (6.3) imply

$$
\begin{aligned}
B[v, v] & :=\int_{Q} A_{1} \nabla v \cdot \nabla v=\left\|\left(A_{1}\right)^{1 / 2} \nabla v\right\|_{L^{2}(Q)}^{2} \geq C\left\|A_{1} \nabla v\right\|_{L^{2}(Q)}^{2} \\
& \geq v\|v\|_{H^{1}(Q)} .
\end{aligned}
$$

Now by the Lax-Milgram lemma, there exists a unique solution $w \in V(\varkappa)^{\perp}$ to the problem

$$
B[w, \varphi]=\langle G, \varphi\rangle \quad \forall \varphi \in V(\varkappa)^{\perp},
$$

and hence to (6.1).

(iii) If $w$ satisfies (6.1) and $v \in V(\varkappa)$, then $A_{1} \nabla v=0$ and hence $w+v$ also satisfies (6.1). Assuming further that $w_{1}$ and $w_{2}$ both satisfy (6.1), notice that $v=w_{1}-w_{2}$ is a solution of (6.1) with $G=0$. Finally, setting $\varphi=v$ in (6.5) yields

$$
0=\int_{Q} A_{1} \nabla v \cdot \nabla v=\left\|\left(A_{1}\right)^{1 / 2} \nabla v\right\|_{L^{2}(Q)}^{2},
$$

implying that $\left(A_{1}\right)^{1 / 2} \nabla v=0$ and hence $A_{1} \nabla v=0$, that is one has $v \in$ $V(\varkappa)$. Assuming now that the solutions $w_{1}, w_{2}$ are in $V(\varkappa)^{\perp}$, the difference $v=w_{1}-w_{2}$ belongs to both $V(\varkappa)$ and $V(\varkappa)^{\perp}$ and is therefore zero.

Corollary 6.1. For each $k=1, \ldots, d$, there exists a unique solution $N_{k} \in V^{\perp}$ to the unit-cell problem (5.5). In particular, for all $\theta \in \varepsilon^{-1} Q^{\prime}$ and $c^{(0)} \in \mathbb{C}$, there exists a unique solution $u^{(1)} \in V^{\perp}$ to the problem (5.4), for which the estimate

$$
\left\|u^{(1)}\right\|_{H^{1}(Q)} \leq\left\|N_{k}\right\|_{H^{1}(Q)}\left|\theta_{k}\right|\left|c^{(0)}\right|
$$

holds.

\subsection{Elliptic Estimates}

In our proof of Theorem 4.1 we use the following two statements.

Lemma 6.2. For each $\theta \in \varepsilon^{-1} Q^{\prime}$, let $u_{\theta}^{(0)}=\mathcal{I}\left(c_{\theta}^{(0)}, v_{\theta}^{(0)}\right)$, where $\left(c_{\theta}^{(0)}, v_{\theta}^{(0)}\right)$ is the solution to (5.9) with $F \in L^{2}(Q)$, and let $u_{\theta}^{(1)} \in H_{\#}^{1}(Q)$ be the solution (5.6) to the unit-cell problem (5.4). Then the following estimates hold with some $C>0$ :

$$
\begin{aligned}
\left|c_{\theta}^{(0)}\right| & \leq C\left(1+|\theta|^{2}\right)^{-1}\|F\|_{L^{2}(Q)}, \\
\left\|u_{\theta}^{(0)}\right\|_{H^{1}(Q)} & \leq C\|F\|_{L^{2}(Q)}, \\
\left\|u_{\theta}^{(1)}\right\|_{H^{1}(Q)} & \leq C|\theta|\left(1+|\theta|^{2}\right)^{-1}\|F\|_{L^{2}(Q)} .
\end{aligned}
$$


Proof. Setting $(d, \varphi)=\left(c_{\theta}^{(0)}, v_{\theta}^{(0)}\right)$ in (5.9), and dropping the scripts " $(0)$ " and " $\theta$ " for convenience, yields

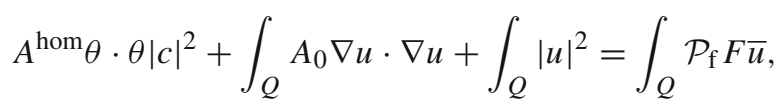

and (6.8) follows by the Cauchy-Schwarz inequality.

Setting $(d, \varphi)=\left(c_{\theta}^{(0)}, 0\right)$ in $(5.9)$ yields

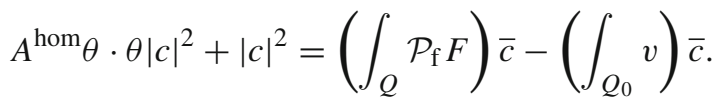

Using the estimate

$$
\begin{aligned}
\left(\int_{Q} \mathcal{P}_{\mathrm{f}} F\right) \bar{c}-\left(\int_{Q_{0}} v\right) \bar{c} & \leq\|F\|_{L^{2}(Q)}|c|+\left|Q_{0}\right|^{1 / 2}\|v\|_{L^{2}(Q)}|c| \\
& \leq\|F\|_{L^{2}(Q)}|c|+\left|Q_{0}\right|^{1 / 2}\|u\|_{L^{2}(Q)}|c|+\left|Q_{0}\right|^{1 / 2}|c|^{2}
\end{aligned}
$$

along with the positivity of $A^{\text {hom }}$ and the bound (6.8), we infer (6.7). The estimate (6.9) is now a direct consequence of (6.7) and (6.6).

Lemma 6.3. For each $\theta \in \varepsilon^{-1} Q^{\prime},|\theta| \geq 1$, let $u_{\varepsilon, \theta}^{(0)}=\mathcal{I}\left(c_{\varepsilon, \theta}^{(0)}, v_{\varepsilon, \theta}^{(0)}\right)$, where the pair $\left(c_{\varepsilon, \theta}^{(0)}, v_{\varepsilon, \theta}^{(0)}\right) \in \mathcal{H}_{0}$ satisfies the identity

$$
\begin{aligned}
& \mathfrak{b}_{\varepsilon, \theta}^{\text {hom }}\left(\left(c_{\varepsilon, \theta}^{(0)}, v_{\varepsilon, \theta}^{(0)}\right),(d, \varphi)\right)+\int_{Q}\left(c_{\varepsilon, \theta}^{(0)}+v_{\varepsilon, \theta}^{(0)}\right) \overline{(d+\varphi)} \\
& \quad=\int_{Q} \mathcal{P}_{\mathrm{f}} \overline{F(d+\varphi)} \forall(d, \varphi) \in \mathcal{H}_{0},
\end{aligned}
$$

with $F \in L^{2}(Q)$. We denote by $u_{\varepsilon, \theta}^{(1)}$ a solution to the unit-cell problem

$$
-\nabla \cdot A_{1} \nabla u_{\varepsilon, \theta}^{(1)}=\mathrm{i} \nabla \cdot A_{1} \theta c_{\varepsilon, \theta}^{(0)}
$$

such that

$$
\int_{Q} A_{1} \theta \cdot \theta u_{\varepsilon, \theta}^{(1)}=0 .
$$

Then the following estimates hold with some $C>0$ :

$$
\begin{aligned}
\left|c_{\varepsilon, \theta}^{(0)}\right| & \leq C(1+|\theta|)^{-2}\|F\|_{L^{2}(Q)}, \\
\left\|u_{\varepsilon, \theta}^{(0)}\right\|_{L^{2}(Q)} & \leq C\|F\|_{L^{2}(Q)}, \\
\left\|(\nabla+\mathrm{i} \varepsilon \theta) v_{\varepsilon, \theta}^{(0)}\right\|_{L^{2}(Q)} & \leq C\|F\|_{L^{2}(Q)}, \\
\left\|u_{\varepsilon, \theta}^{(1)}\right\|_{H^{1}(Q)} & \leq C|\theta|\left(1+|\theta|^{2}\right)^{-1}\|F\|_{L^{2}(Q)} .
\end{aligned}
$$


Proof. Taking the unique solution $w_{\varepsilon, \theta} \in V^{\perp}$ to the problem

$$
-\nabla \cdot A_{1} \nabla w_{\varepsilon, \theta}=\mathrm{i} \nabla \cdot A_{1} \theta c_{\varepsilon, \theta}^{(0)},
$$

we find by Corollary 6.1 that

$$
\left\|w_{\varepsilon, \theta}\right\|_{H^{1}(Q)} \leq C|\theta|\left|c_{\varepsilon, \theta}^{(0)}\right| \text {. }
$$

Denoting $u_{\varepsilon, \theta}^{(1)}=w_{\varepsilon, \theta}-\left(\int_{Q} A_{1} \theta \cdot \theta\right)^{-1} \int_{Q} A_{1} \theta \cdot \theta w_{\varepsilon, \theta}$, it is clear that (6.11) holds. By the properties of boundedness and ellipticity of $A_{1}$ we find that

$$
\left(\int_{Q} A_{1} \theta \cdot \theta\right)^{-1} \int_{Q} A_{1} \theta \cdot \theta w_{\varepsilon, \theta} \leq C\left\|w_{\varepsilon, \theta}\right\|_{L^{2}(Q)} .
$$

In particular, the estimate

$$
\left\|u_{\varepsilon, \theta}^{(1)}\right\|_{H^{1}(Q)} \leq C|\theta|\left|c_{\varepsilon, \theta}^{(0)}\right|
$$

holds.

Inequalities (6.12)-(6.15) are now shown by appropriately modifying the proof of Lemma 6.2.

Lemma 6.4. Let $\theta \in \varepsilon^{-1} Q^{\prime}$, and let $F_{\theta}$ be given by (5.8). There exists a function $R_{\theta} \in H_{\#}^{1}(Q)$ satisfying

$$
-\nabla \cdot A_{1} \nabla R_{\theta}=F_{\theta}
$$

such that

$$
\left\|R_{\theta}\right\|_{H^{1}(Q)} \leq C\|F\|_{L^{2}(Q)}
$$

for some constant $C>0$ independent of $\varepsilon, \theta$.

Proof. The functions $u^{(0)}$ and $u^{(1)}$ are chosen so that $F_{\theta}$ satisfies the solvability condition for the equation (5.7), thus the existence of a solution $u^{(2)}$ is guaranteed by Lemma 6.1 . Denoting by $R_{\theta}$ the unique part in $V^{\perp}$ of any such solution, that is letting $R_{\theta} \in V^{\perp}$ be such that

$$
\int_{Q} A_{1} \nabla R_{\theta} \cdot \nabla \varphi=\left\langle F_{\theta}, \varphi\right\rangle \quad \forall \varphi \in H_{\#}^{1}(Q),
$$

we find, by choosing $\varphi=R_{\theta}$ in (6.17) and using the assumptions on $A_{1}$, that

$$
\left\|A_{1} \nabla R_{\theta}\right\|_{L^{2}(Q)}^{2} \leq\left\|A_{1}^{1 / 2}\right\|_{L^{\infty}(Q)}^{2}\left\|A_{1}^{1 / 2} \nabla R_{\theta}\right\|_{L^{2}(Q)}^{2} \leq C\left\|F_{\theta}\right\|_{H_{\#}^{-1}(Q)}\left\|R_{\theta}\right\|_{H_{\#}^{1}(Q)},
$$

where $A_{1}^{1 / 2}$ is the square root of the matrix $A_{1}$. Due to Lemma 6.1(i), it remains to show that

$$
\left\|F_{\theta}\right\|_{H_{\#}^{-1}(Q)} \leq C\|F\|_{L^{2}(Q)}
$$


for some constant $C$. This can be seen by Lemma 6.2 and by noting, for $\theta \in \varepsilon^{-1} Q^{\prime}$, that

$$
\begin{aligned}
& \left|\left\langle F_{\theta}, \varphi\right\rangle\right| \\
& \quad=\left|\int_{Q} F \bar{\varphi}-\mathrm{i} A_{1} u_{\theta}^{(1)} \theta \cdot \overline{\nabla \varphi}+\mathrm{i} \theta \cdot A_{1} \nabla u_{\theta}^{(1)} \bar{\varphi}-A_{0} \nabla u_{\theta}^{(0)} \cdot \overline{\nabla \varphi}-\theta \cdot A_{1} \theta c_{\theta}^{(0)} \bar{\varphi}-u_{\theta}^{(0)} \bar{\varphi}\right| \\
& \quad \leq C\left(\|F\|_{L^{2}(Q)}+|\theta|\left\|u_{\theta}^{(1)}\right\|_{H^{1}(Q)}+\left\|u_{\theta}^{(0)}\right\|_{H^{1}(Q)}+|\theta|^{2}\left|c_{\theta}^{(0)}\right|\right)\|\varphi\|_{H^{1}(Q)}
\end{aligned}
$$

for all $\varphi \in H_{\#}^{1}(Q)$.

Lemma 6.5. For each $\varepsilon>0, \theta \neq 0$, consider $H_{\varepsilon, \theta} \in H_{\#}^{-1}(Q)$ such that $\left\langle H_{\varepsilon, \theta}, \varphi\right\rangle=0$ for all $\varphi \in H_{0}^{1}\left(Q_{0}\right)$. Then there exists a solution $R_{\varepsilon, \theta} \in H_{\#}^{1}(Q)$ to the problem

$$
-(\nabla+\mathrm{i} \varepsilon \theta) \cdot A_{1}(\nabla+\mathrm{i} \varepsilon \theta) R_{\varepsilon, \theta}=H_{\varepsilon, \theta}
$$

that satisfies the estimate

$$
\left\|R_{\varepsilon, \theta}\right\|_{H^{1}(Q)} \leq C\left[\frac{1}{|\varepsilon \theta|}\left\|H_{\varepsilon, \theta}-\left\langle H_{\varepsilon, \theta}, 1\right\rangle\right\|_{H_{\#}^{-1}(Q)}+\frac{1}{|\varepsilon \theta|^{2}}\left|\left\langle H_{\varepsilon, \theta}, 1\right\rangle\right|\right]
$$

for some constant $C>0$ independent of $\varepsilon, \theta$.

Proof. For $\varkappa \neq 0, V(\varkappa)=H_{0}^{1}\left(Q_{0}\right)$, see (6.2). By Lemma 6.1, the assumption $\left\langle H_{\varepsilon, \theta}, \varphi\right\rangle=0$ for all $\varphi \in H_{0}^{1}\left(Q_{0}\right)$ implies that there exists a unique weak solution $w_{\varepsilon, \theta} \in V^{\perp}(\varepsilon \theta)$ to the problem

$$
-\nabla \cdot A_{1} \nabla w_{\varepsilon, \theta}(y)=\exp (\mathrm{i} \varepsilon \theta \cdot y) H_{\varepsilon, \theta}(y), \quad y \in Q,
$$

and

$$
\left\|w_{\varepsilon, \theta}\right\|_{H^{1}(Q)} \leq \frac{C}{|\varepsilon \theta|}\left\|A_{1} \nabla w_{\varepsilon, \theta}\right\|_{L^{2}(Q)}
$$

As $w_{\varepsilon, \theta} \in H_{\varepsilon \theta}^{1}(Q)$, the function $r_{\varepsilon, \theta}(y):=\exp (-\mathrm{i} \varepsilon \theta \cdot y) w_{\varepsilon, \theta}(y)$ is an element of $H_{\#}^{1}(Q)$ and satisfies the identity

$$
\int_{Q} A_{1}(\nabla+\mathrm{i} \varepsilon \theta) r_{\varepsilon, \theta} \cdot \overline{(\nabla+\mathrm{i} \varepsilon \theta) \varphi}=\left\langle H_{\varepsilon, \theta}, \varphi\right\rangle \quad \forall \varphi \in H_{\#}^{1}(Q),
$$

and by (6.19) we find that

$$
\left\|r_{\varepsilon, \theta}\right\|_{H^{1}(Q)} \leq \frac{C}{|\varepsilon \theta|^{2}}\left\|H_{\varepsilon, \theta}\right\|_{H_{\#}^{-1}(Q)} .
$$

The estimate (6.18) can be viewed as a refined version of (6.21) by writing $r_{\varepsilon, \theta}=$ $s_{\varepsilon, \theta}+t_{\varepsilon, \theta}$, where $s_{\varepsilon, \theta}, t_{\varepsilon, \theta}$ are the solutions to (6.20) for the right-hand sides $H_{\varepsilon, \theta}-$ $\left\langle H_{\varepsilon, \theta}, 1\right\rangle \psi$ and $\left\langle H_{\varepsilon, \theta}, 1\right\rangle \psi$ respectively, for any chosen $\psi \in L^{2}(Q),\left.\psi\right|_{Q_{0}}=0$, 
$\int_{Q} \psi=1$. We then argue that in order to prove the theorem it is sufficient to ensure that there exists a function $R_{\varepsilon, \theta}$ solving (6.20) which satisfies the bound

$$
\left\|R_{\varepsilon, \theta}\right\|_{H^{1}(Q)} \leq \frac{C}{|\varepsilon \theta|}\left\|H_{\varepsilon, \theta}\right\|_{H_{\#}^{-1}(Q)}
$$

for the class of $H_{\varepsilon, \theta} \in H_{\#}^{-1}(Q)$ such that $\left\langle H_{\varepsilon, \theta}, 1\right\rangle=0$. Indeed, one has

$$
\left\langle H_{\varepsilon, \theta}-\left\langle H_{\varepsilon, \theta}, 1\right\rangle \psi, \varphi\right\rangle=0, \quad\left\langle\left\langle H_{\varepsilon, \theta}, 1\right\rangle \psi, \varphi\right\rangle=0 \quad \forall \varphi \in H_{0}^{1}\left(Q_{0}\right),
$$

and

$$
\left\langle H_{\varepsilon, \theta}-\left\langle H_{\varepsilon, \theta}, 1\right\rangle \psi, 1\right\rangle=\left\langle H_{\varepsilon, \theta}, 1\right\rangle-\left\langle H_{\varepsilon, \theta}, 1\right\rangle \int_{Q} \psi=0
$$

Let $w_{\varepsilon, \theta}, r_{\varepsilon, \theta}$ be as above for a given $H_{\varepsilon, \theta} \in H_{\#}^{-1}(Q)$ such that $\left\langle H_{\varepsilon, \theta}, 1\right\rangle=0$. Denoting by $R_{\varepsilon, \theta}$ an extension of $r_{\varepsilon, \theta}$ such that

$$
\begin{aligned}
& R_{\varepsilon, \theta}=r_{\varepsilon, \theta} \quad \text { in } Q_{1}, \\
& \left\|\nabla R_{\varepsilon, \theta}\right\|_{L^{2}(Q)} \leq C\left\|\nabla r_{\varepsilon, \theta}\right\|_{L^{2}\left(Q_{1}\right)},
\end{aligned}
$$

it is clear that $R_{\varepsilon, \theta}$ also satisfies (6.20) with $r_{\varepsilon, \theta}$ replaced by $R_{\varepsilon, \theta}$. We next show that $R_{\varepsilon, \theta}$ satisfies the inequality (6.22).

Substituting $\varphi \equiv 1$ in (6.20), and recalling that $\left\langle H_{\varepsilon, \theta}, 1\right\rangle=0$, we infer that

$$
\int_{Q} R_{\varepsilon, \theta}=\left(\int_{Q} \varepsilon^{2} A_{1} \theta \cdot \theta\right)^{-1}\left(-\int_{Q} \varepsilon^{2} A_{1} \theta \cdot \theta\left(R_{\varepsilon, \theta}-\int_{Q} R_{\varepsilon, \theta}\right)+\mathrm{i} \varepsilon \int_{Q} A_{1} \nabla R_{\varepsilon, \theta} \cdot \theta\right),
$$

and hence

$$
\left|\int_{Q} R_{\varepsilon, \theta}\right| \leq \frac{C}{|\varepsilon \theta|^{2}}\left(|\varepsilon \theta|^{2}\left\|R_{\varepsilon, \theta}-\int_{Q} R_{\varepsilon, \theta}\right\|_{L^{2}(Q)}+|\varepsilon \theta|\left\|\nabla R_{\varepsilon, \theta}\right\|_{L^{2}\left(Q_{1}\right)}\right) .
$$

In particular, by (6.24) and the standard Poincaré inequality, it follows that

$$
\left|\int_{Q} R_{\varepsilon, \theta}\right| \leq \frac{C}{|\varepsilon \theta|}\left\|\nabla r_{\varepsilon, \theta}\right\|_{L^{2}\left(Q_{1}\right)} .
$$

Therefore, by (6.23)-(6.25) we find that

$$
\left\|R_{\varepsilon, \theta}\right\|_{H^{1}(Q)} \leq C\left(\left|\int_{Q} R_{\varepsilon, \theta}\right|^{2}+\int_{Q}\left|\nabla R_{\varepsilon, \theta}\right|^{2}\right)^{1 / 2} \leq \frac{C}{|\varepsilon \theta|}\left\|\nabla r_{\varepsilon, \theta}\right\|_{L^{2}\left(Q_{1}\right)} .
$$

To prove (6.22) it now remains to show that

$$
\left\|\nabla r_{\varepsilon, \theta}\right\|_{L^{2}\left(Q_{1}\right)} \leq C\left\|H_{\varepsilon, \theta}\right\|_{H_{\#}^{-1}(Q)}
$$

for some constant $C>0$. By virtue of the inequality $\left\|r_{\varepsilon, \theta}\right\|_{L^{2}(Q)} \leq\left\|w_{\varepsilon, \theta}\right\|_{H^{1}(Q)}$ and (6.19) we find that 


$$
\begin{aligned}
& \left\|\nabla r_{\varepsilon, \theta}\right\|_{L^{2}\left(Q_{1}\right)} \leq\left\|(\nabla+\mathrm{i} \varepsilon \theta) r_{\varepsilon, \theta}\right\|_{L^{2}\left(Q_{1}\right)} \\
& \quad+|\varepsilon \theta|\left\|r_{\varepsilon, \theta}\right\|_{L^{2}\left(Q_{1}\right)} \leq C\left\|(\nabla+\mathrm{i} \varepsilon \theta) r_{\varepsilon, \theta}\right\|_{L^{2}\left(Q_{1}\right)}
\end{aligned}
$$

Further, substituting $\varphi=R_{\varepsilon, \theta}$ in (6.20) and recalling (6.23) yields

$$
\begin{aligned}
& \int_{Q} A_{1}(\nabla+\mathrm{i} \varepsilon \theta) r_{\varepsilon, \theta} \cdot \overline{(\nabla+\mathrm{i} \varepsilon \theta) r_{\varepsilon, \theta}}=\left\langle H_{\varepsilon, \theta}, R_{\varepsilon, \theta}\right\rangle \\
& =\left\langle H_{\varepsilon, \theta}, R_{\varepsilon, \theta}-\int_{Q} R_{\varepsilon, \theta}\right\rangle+\left\langle H_{\varepsilon, \theta}, \int_{Q} R_{\varepsilon, \theta}\right\rangle \\
& =\left\langle H_{\varepsilon, \theta}, R_{\varepsilon, \theta}-\int_{Q} R_{\varepsilon, \theta}\right\rangle \leq C\left\|H_{\varepsilon, \theta}\right\|_{H_{\#}^{-1}(Q)}\left\|\nabla R_{\varepsilon, \theta}\right\|_{L^{2}(Q)} \\
& \quad \leq C\left\|H_{\varepsilon, \theta}\right\|_{H_{\#}^{-1}(Q)}\left\|\nabla r_{\varepsilon, \theta}\right\|_{L^{2}\left(Q_{1}\right)} .
\end{aligned}
$$

The last equality above follows from the assumption that $\left\langle H_{\varepsilon, \theta}, 1\right\rangle=0$. Finally, inequalities (6.27) and (6.28) imply (6.26).

\section{Proof of the Main Result}

In terms of the notation introduced in Sections 3 and 4, proving Theorem 4.1 is equivalent to showing that there exists a constant $C>0$ independent of $\theta$ and $\varepsilon$ such that

$$
\left\|\left(\mathcal{B}_{\varepsilon, \theta}+1\right)^{-1}-\mathcal{I}\left(\mathcal{B}_{\varepsilon, \theta}^{\mathrm{hom}}+1\right)^{-1} \mathcal{I}^{-1} \mathcal{P}_{\mathrm{f}}\right\|_{L^{2}(Q) \rightarrow L^{2}(Q)} \leq C \varepsilon .
$$

This fact is a consequence of the following theorem.

Theorem 7.1. For each $\varepsilon>0, \theta \in \varepsilon^{-1} Q^{\prime}$, let $u_{\theta}^{\varepsilon}$ be the solution to (3.1) and let $u_{\varepsilon, \theta}^{(0)}:=c_{\varepsilon, \theta}^{(0)}+v_{\varepsilon, \theta}^{(0)}$ where the pair $\left(c_{\varepsilon, \theta}^{(0)}, v_{\varepsilon, \theta}^{(0)}\right) \in \mathcal{H}_{0}$ satisfies the identity (6.10).

Then there exists a constant $C>0$ independent of $\theta$ and $\varepsilon$ such that

$$
\left\|u_{\theta}^{\varepsilon}-u_{\varepsilon, \theta}^{(0)}\right\|_{L^{2}(Q)} \leq C \varepsilon\|F\|_{L^{2}(Q)} .
$$

Proof. To prove the result we consider $\theta \in \varepsilon^{-1} Q^{\prime}$ in two regions.

Case 1: $|\theta| \leq 1$. Let $U_{\varepsilon, \theta}^{(1)}=u_{\theta}^{(0)}+\varepsilon u_{\theta}^{(1)}+\varepsilon^{2} R_{\theta}$, where $u_{\theta}^{(0)}, u_{\theta}^{(1)}$ and $R_{\theta}$ are given by (5.9), (5.4) and Lemma 6.4, respectively. Due to the fact that the functions $u_{\theta}^{(0)}$ and $u_{\varepsilon, \theta}^{(0)}$ are $\varepsilon$-close in $L^{2}(Q)$ uniformly in $\theta$ for $|\theta| \leq 1$ (cf. Lemma 5.1), it is sufficient to prove that

$$
\left\|u_{\theta}^{\varepsilon}-U_{\varepsilon, \theta}^{(1)}\right\|_{L^{2}(Q)} \leq C \varepsilon\|F\|_{L^{2}(Q)} .
$$

By direct calculation we find that the difference $z_{\varepsilon, \theta}^{(1)}:=u_{\theta}^{\varepsilon}-U_{\varepsilon, \theta}^{(1)}$ is the $H_{\#}^{1}(Q)$ solution of the equation

$$
-\varepsilon^{-2}(\nabla+\mathrm{i} \varepsilon \theta) \cdot A^{\varepsilon}(\nabla+\mathrm{i} \varepsilon \theta) z_{\varepsilon, \theta}^{(1)}+z_{\varepsilon, \theta}^{(1)}=F_{\varepsilon, \theta}^{(1)},
$$


where the coefficients for the non-positive powers of $\varepsilon$ have cancelled due to the construction of $U_{\varepsilon, \theta}^{(1)}$. The right-hand side $F_{\varepsilon, \theta}^{(1)} \in H_{\#}^{-1}(Q)$ of (7.1) takes the form

$$
F_{\varepsilon, \theta}^{(1)}:=\sum_{n=1}^{4} \varepsilon^{n} T_{\theta}^{(n)},
$$

where

$$
\begin{aligned}
T_{\theta}^{(1)}:= & \mathrm{i}\left(\nabla \cdot A_{1} \theta+\theta \cdot A_{1} \nabla\right) R_{\theta}+\left(\nabla \cdot A_{0} \nabla-\theta \cdot A_{1} \theta-I\right) u_{\theta}^{(1)} \\
& +\mathrm{i}\left(\nabla \cdot A_{0} \theta+\theta \cdot A_{0} \nabla\right) u_{\theta}^{(0)}, \\
T_{\theta}^{(2)}:= & \left(\nabla \cdot A_{0} \nabla-\theta \cdot A_{1} \theta-I\right) R_{\theta}+\mathrm{i}\left(\nabla \cdot A_{0} \theta+\theta \cdot A_{0} \nabla\right) u_{\theta}^{(1)}-\theta \cdot A_{0} \theta u_{\theta}^{(0)}, \\
T_{\theta}^{(3)}:= & \mathrm{i}\left(\nabla \cdot A_{0} \theta+\theta \cdot A_{0} \nabla\right) R_{\theta}-\theta \cdot A_{0} \theta u_{\theta}^{(1)}, \\
T_{\theta}^{(4)}:= & -\theta \cdot A_{0} \theta R_{\theta}
\end{aligned}
$$

are elements of $H_{\#}^{-1}(Q)$.

A straightforward calculation shows that equations (7.2)-(7.5) with the inequalities (6.8), (6.9) and (6.16) imply the bound

$$
\left\|F_{\varepsilon, \theta}^{(1)}\right\|_{H_{\#}^{-1}(Q)} \leq C \varepsilon\|F\|_{L^{2}(Q)} .
$$

Hence, the required inequality

$$
\left\|z_{\varepsilon, \theta}^{(1)}\right\|_{L^{2}(Q)} \leq C \varepsilon\|F\|_{L^{2}(Q)}
$$

follows.

Case 2: $|\theta| \geq 1$. Let $U_{\varepsilon, \theta}^{(2)}=u_{\varepsilon, \theta}^{(0)}+\varepsilon u_{\varepsilon, \theta}^{(1)}+\varepsilon^{2} R_{\varepsilon, \theta}$, where $u_{\varepsilon, \theta}^{(1)}$ is defined in Lemma 6.3 and $R_{\varepsilon, \theta}$ is given by Lemma 6.5 for the right-hand side

$$
\begin{aligned}
H_{\varepsilon, \theta}:= & F+\mathrm{i}\left(\nabla \cdot A_{1} \theta+\theta \cdot A_{1} \nabla\right) u_{\varepsilon, \theta}^{(1)}-\varepsilon \theta \cdot A_{1} \theta u_{\varepsilon, \theta}^{(1)} \\
& +(\nabla+\mathrm{i} \varepsilon \theta) \cdot A_{0}(\nabla+\mathrm{i} \varepsilon \theta) v_{\varepsilon, \theta}^{(0)}-\theta \cdot A_{1} \theta c_{\varepsilon, \theta}^{(0)}-u_{\varepsilon, \theta}^{(0)} .
\end{aligned}
$$

Notice that the following inequalities hold

$$
\left\|H_{\varepsilon, \theta}-\left\langle H_{\varepsilon, \theta}, 1\right\rangle\right\|_{H_{\#}^{-1}(Q)} \leq C\|F\|_{L^{2}(Q)}, \quad\left|\left\langle H_{\varepsilon, \theta}, 1\right\rangle\right| \leq C|\varepsilon \theta|\|F\|_{L^{2}(Q)},
$$

for some constant $C>0$ independent of $\varepsilon$ and $\theta$. These follow from Lemma 6.3 and the estimates

$$
\begin{aligned}
\left|\left\langle H_{\varepsilon, \theta}, \varphi\right\rangle\right|= & \mid \int_{Q}\left(F \bar{\varphi}-\mathrm{i} A_{1} u_{\varepsilon, \theta}^{(1)} \theta \cdot \overline{\nabla \varphi}+\mathrm{i} \theta \cdot A_{1} \nabla u_{\varepsilon, \theta}^{(1)} \bar{\varphi}-\varepsilon \theta \cdot A_{1} \theta u_{\varepsilon, \theta}^{(1)} \bar{\varphi}\right) \\
& -\int_{Q}\left(A_{0}(\nabla+\mathrm{i} \varepsilon \theta) v_{\varepsilon, \theta}^{(0)} \cdot \overline{(\nabla+\mathrm{i} \varepsilon \theta) \varphi}+\theta \cdot A_{1} \theta c_{\varepsilon, \theta}^{(0)} \bar{\varphi}+u_{\varepsilon, \theta}^{(0)} \bar{\varphi}\right) \mid
\end{aligned}
$$




$$
\begin{aligned}
& \leq C\left(\|F\|_{L^{2}(Q)}+|\theta|\left\|u_{\varepsilon, \theta}^{(1)}\right\|_{H^{1}(Q)}\right. \\
& \left.+\left\|(\nabla+\mathrm{i} \varepsilon \theta) v_{\varepsilon, \theta}^{(0)}\right\|_{L^{2}\left(Q_{0}\right)}+|\theta|^{2}\left|c_{\varepsilon, \theta}^{(0)}\right|+\left\|u_{\varepsilon, \theta}^{(0)}\right\|_{L^{2}(Q)}\right)\|\varphi\|_{H^{1}(Q)}
\end{aligned}
$$

and

$$
\left|\left\langle H_{\varepsilon, \theta}, 1\right\rangle\right|=\left|\int_{Q} A_{0}(\nabla+\mathrm{i} \varepsilon \theta) v_{\varepsilon, \theta}^{(0)} \cdot \overline{\mathrm{i} \varepsilon \theta}\right| \leq C|\varepsilon \theta|\left\|(\nabla+\mathrm{i} \varepsilon \theta) v_{\varepsilon, \theta}^{(0)}\right\|_{L^{2}\left(Q_{0}\right)} .
$$

The above inequalities, in combination with (6.18), imply that $R_{\varepsilon, \theta} \in H_{\#}^{1}(Q)$ can be chosen so that

$$
\left\|R_{\varepsilon, \theta}\right\|_{H^{1}(Q)} \leq C \frac{1}{|\varepsilon \theta|}\|F\|_{L^{2}(Q)} .
$$

By direct calculation we find that the "error" $z_{\varepsilon, \theta}^{(2)}:=u_{\theta}^{\varepsilon}-U_{\varepsilon, \theta}^{(2)}$ is the $H_{\#}^{1}(Q)$ solution of the equation

$$
-\varepsilon^{-2}(\nabla+\mathrm{i} \varepsilon \theta) \cdot A^{\varepsilon}(\nabla+\mathrm{i} \varepsilon \theta) z_{\varepsilon, \theta}^{(2)}+z_{\varepsilon, \theta}^{(2)}=F_{\varepsilon, \theta}^{(2)},
$$

where the coefficients for the non-positive powers of $\varepsilon$ have cancelled due to the construction of $U_{\varepsilon, \theta}^{(2)}$. In the above equation the right-hand side $F_{\varepsilon, \theta}^{(2)} \in H_{\#}^{-1}(Q)$ is given by

$$
F_{\varepsilon, \theta}^{(2)}:=\sum_{n=1}^{4} \varepsilon^{n} S_{\theta}^{(n)}
$$

where

$$
\begin{aligned}
S_{\theta}^{(1)} & :=\left(\nabla \cdot A_{0} \nabla-I\right) u_{\varepsilon, \theta}^{(1)}+\mathrm{i}\left(\nabla \cdot A_{0} \theta+\theta \cdot A_{0} \nabla\right) c_{\varepsilon, \theta}^{(0)}, \\
S_{\theta}^{(2)} & :=\left(\nabla \cdot A_{0} \nabla-I\right) R_{\varepsilon, \theta}+\mathrm{i}\left(\nabla \cdot A_{0} \theta+\theta \cdot A_{0} \nabla\right) u_{\varepsilon, \theta}^{(1)}-\theta \cdot A_{0} \theta c_{\varepsilon, \theta}^{(0)}, \\
S_{\theta}^{(3)} & :=\mathrm{i}\left(\nabla \cdot A_{0} \theta+\theta \cdot A_{0} \nabla\right) R_{\varepsilon, \theta}-\theta \cdot A_{0} \theta u_{\varepsilon, \theta}^{(1)}, \\
S_{\theta}^{(4)} & :=-\theta \cdot A_{0} \theta R_{\varepsilon, \theta}
\end{aligned}
$$

are elements of $H_{\#}^{-1}(Q)$. Equations (7.7)-(7.10) together with inequalities (6.12), (6.15) and (7.6) imply that

$$
\left\|F_{\varepsilon, \theta}^{(2)}\right\|_{H_{\#}^{-1}(Q)} \leq C \varepsilon\|F\|_{L^{2}(Q)} .
$$

Therefore, the bound

$$
\left\|z_{\varepsilon, \theta}^{(2)}\right\|_{H^{1}(Q)} \leq C \varepsilon\|F\|_{L^{2}(Q)}
$$

holds, and the result follows. 


\section{The Outer Expansion and Principal Term for $\mathcal{B}_{\varepsilon, \theta}^{\text {hom }}$ in the Outer Region}

$$
|\theta| \geq \varepsilon^{-1 / 2}
$$

For fixed $\varkappa \neq 0$ we shall study the asymptotics of the following problem: find $w_{\varepsilon, \varkappa} \in H_{\varkappa}^{1}(Q)$ such that

$$
-\nabla \cdot\left(\varepsilon^{-2} A_{1}+A_{0}\right) \nabla w_{\varepsilon, \varkappa}+w_{\varepsilon, \varkappa}=F, \quad F \in L^{2}(Q) .
$$

Let us consider an asymptotic expansion for the solution to the above problem of the form

$$
w_{\varepsilon, \varkappa}=\sum_{n=0}^{\infty} \varepsilon^{2 n} w_{\varkappa}^{(n)}, \quad w_{\varkappa}^{(n)} \in H_{\varkappa}^{1}(Q), \quad n=0,1,2, \ldots
$$

Substituting (8.2) in (8.1) and comparing the coefficients in front of $\varepsilon^{-2}$ on both sides of the resulting equation yields

$$
\nabla \cdot A_{1} \nabla w_{\varkappa}^{(0)}=0
$$

that is $w_{\varkappa}^{(0)} \in V(\varkappa)$ or, equivalently, $w_{\varkappa}^{(0)} \in H_{0}^{1}\left(Q_{0}\right)$, see (6.2). Further, comparing the coefficients in front of $\varepsilon^{0}$ yields

$$
-\nabla \cdot A_{1} \nabla w_{\varkappa}^{(1)}=F+\nabla \cdot A_{0} \nabla w_{\varkappa}^{(0)}-w_{\varkappa}^{(0)} .
$$

The existence of a solution to (8.3) is guaranteed by Lemma 6.1 if and only if $w_{\varkappa}^{(0)}$ satisfies the identity

$$
\int_{Q_{0}}\left(A_{0} \nabla w_{\varkappa}^{(0)} \cdot \nabla \varphi+w_{\varkappa}^{(0)} \varphi\right)=\langle F, \varphi\rangle \quad \forall \varphi \in H_{0}^{1}\left(Q_{0}\right) .
$$

Existence and uniqueness of $w_{\varkappa}^{(0)}$ is implied by the ellipticity of $A_{0}$ in $Q_{0}$ and standard ellipticity estimates give the inequality

$$
\left\|w_{\varkappa}^{(0)}\right\|_{H_{0}^{1}\left(Q_{0}\right)} \leq C\|F\|_{L^{2}(Q)} .
$$

Furthermore, by Lemma $6.1,(8.5)$ and (8.3) the unique part in $V(\varkappa)^{\perp}$ of such a solution satisfies the inequality

$$
\left\|P_{V(\varkappa)^{\perp}} w_{\varkappa}^{(1)}\right\|_{H^{1}(Q)} \leq \frac{C}{|\varkappa|^{2}}\|F\|_{L^{2}(Q)},
$$

for some constant $C$ independent of $\varkappa$. Comparing the terms with $\varepsilon^{2 n}$, for $n \geq 1$, yields

$$
-\nabla \cdot A_{1} \nabla w_{\varkappa}^{(n+1)}=\nabla \cdot A_{0} \nabla w_{\varkappa}^{(n)}-w_{\varkappa}^{(n)} .
$$

The existence of a solution to (8.7) is guaranteed by requiring that $P_{V(\varkappa)} w_{\varkappa}^{(n)}$ satisfies the identity 


$$
\begin{aligned}
& \int_{Q_{0}}\left(A_{0} \nabla P_{V(\varkappa)} w_{\varkappa}^{(n)} \cdot \nabla \varphi+P_{V(\varkappa)} w_{\varkappa}^{(n)} \varphi\right) \\
& =-\int_{Q_{0}}\left(A_{0} \nabla P_{V(\varkappa)^{\perp}} w_{\varkappa}^{(n)} \cdot \nabla \varphi+P_{V(\varkappa)^{\perp}} w_{\varkappa}^{(n)} \varphi\right) \quad \forall \varphi \in H_{0}^{1}\left(Q_{0}\right) .
\end{aligned}
$$

Equation (8.8) implies

$$
\left\|P_{V(\varkappa)} w_{\varkappa}^{(n)}\right\|_{H^{1}(Q)} \leq C\left\|P_{V(\varkappa)^{\perp}} w_{\varkappa}^{(n)}\right\|_{H^{1}(Q)}
$$

for some constant $C$. Therefore, by Lemma 6.1, (8.9) and (8.7), there exists a constant $C>0$ independent of $\varkappa$ such that

$$
\left\|P_{V(\varkappa)^{\perp}} w_{\varkappa}^{(n+1)}\right\|_{H^{1}(Q)} \leq \frac{C}{|\varkappa|^{2}}\left\|P_{V(\varkappa)^{\perp}} w_{\varkappa}^{(n)}\right\|_{H^{1}(Q)} .
$$

In particular, by recalling (8.6) we find that

$$
\left\|w_{\varkappa}^{(n)}\right\|_{H^{1}(Q)} \leq \frac{C}{|\varkappa|^{2 n}}\|F\|_{L^{2}(Q)} .
$$

Now constructing the function

$$
U_{\varepsilon, \varkappa}^{(N)}=\sum_{n=0}^{N} \varepsilon^{2 n} w_{\varkappa}^{(n)} \in H_{\varkappa}^{1}(Q),
$$

we have the following result.

Theorem 8.1. Let $w_{\varepsilon, \varkappa}$ be the solution to (8.1). Then for any positive integer $N$ there exists a constant $C_{N}>0$ independent of $\varkappa$ and $\varepsilon$ such that

$$
\left\|w_{\varepsilon, \varkappa}-U_{\varepsilon, \varkappa}^{(N)}\right\|_{H^{1}(Q)} \leq C_{N}\left(\frac{\varepsilon}{|\varkappa|}\right)^{2(N+1)}\|F\|_{L^{2}(Q)} .
$$

In particular,

$$
\left\|w_{\varepsilon . \varkappa}-w_{\varkappa}^{(0)}\right\|_{H^{1}(Q)} \leq C_{1}\left(\frac{\varepsilon}{|\varkappa|}\right)^{2}\|F\|_{L^{2}(Q)} .
$$

Proof. Substituting $U_{\varepsilon, \varkappa}^{(N+1)}$ in to (8.1) and equating powers of $\varepsilon$ yields

$$
\begin{aligned}
& -\nabla \cdot\left(\varepsilon^{-2} A_{1}+A_{0}\right) \nabla\left(w_{\varepsilon, \varkappa}-U_{\varepsilon, \varkappa}^{(N+1)}\right)+w_{\varepsilon, \varkappa}-U_{\varepsilon, \varkappa}^{(N+1)} \\
& =\varepsilon^{2(N+1)}\left(\nabla \cdot A_{0} \nabla w_{\varkappa}^{(N+1)}-w_{\varkappa}^{(N+1)}\right) .
\end{aligned}
$$

Using (8.10) and the standard ellipticity estimates results in an estimate similar to $(8.11)$ for the difference $w_{\varepsilon, \varkappa}-U_{\varepsilon, \varkappa}^{(N+1)}$. Finally, noticing that $U_{\varepsilon, \varkappa}^{(N)}=U_{\varepsilon, \varkappa}^{(N+1)}-$ $\varepsilon^{2(N+1)} w_{\varkappa}^{(N+1)}$ and once again employing (8.10) yields the required estimate (8.11). 
Denote by $[g]$ the multiplication operator for a given function $g$, and denote by $\mathcal{B}_{0}$ the operator associated with the problem (8.4) such that $w_{\varkappa}^{(0)}=\left(\mathcal{B}_{0}+I\right)^{-1} \mathcal{P}_{0} F$, where $\mathcal{P}_{0}$ is the orthogonal projection of $L^{2}(Q)$ onto $H_{0}^{1}\left(Q_{0}\right)$. Theorem $8.1 \mathrm{implies}$ that that $\mathcal{B}_{0}$ is $\varepsilon$-close to $\mathcal{B}_{\varepsilon, \theta}^{\text {hom }}$ in the region $|\theta| \geq \varepsilon^{-1 / 2}$, in the following sense.

Corollary 8.1. There is a constant $C>0$ such that the estimate

$$
\left\|\left[\mathrm{e}^{-\mathrm{i} \varepsilon \theta \cdot}\right]\left(\mathcal{B}_{0}+I\right)^{-1} \mathcal{P}_{0}\left[\mathrm{e}^{\mathrm{i} \varepsilon \theta \cdot}\right]-\mathcal{I}\left(\mathcal{B}_{\varepsilon, \theta}^{\mathrm{hom}}+I\right)^{-1} \mathcal{I}^{-1} \mathcal{P}_{\mathrm{f}}\right\|_{L^{2}(Q)} \leq C \varepsilon,
$$

holds for all $\theta \in \varepsilon^{-1} Q^{\prime}$ such that $|\theta| \geq \varepsilon^{-1 / 2}$.

Corollary 8.1 and Theorem 7.1 imply that in the region $|\varkappa| \geq \varepsilon^{1 / 2}$ the term $w_{\varkappa}^{(0)}$ is the principal term in the approximation to $w_{\varepsilon, \varkappa}(y)=\exp (\mathrm{i} \varkappa \cdot y) u_{\varepsilon^{-1} \varkappa}^{\varepsilon}(y)$, $y \in Q$, in the "slow" variable $\varkappa$. Furthermore, Lemma 5.1 states that in the region $|\theta| \leq 1$ the function $u_{\theta}^{(0)}=\mathcal{I}\left(c_{\theta}^{(0)}, v_{\theta}^{(0)}\right)$ is the principal term in the approximation to $u_{\theta}^{\varepsilon}$ in the "fast" variable $\theta=\varkappa / \varepsilon$. This leads to the presence of a boundary layer in the Bloch space in the region $1 \leq|\theta| \leq \varepsilon^{-1 / 2}$, where neither the "outer" operator $\mathcal{B}_{0}$ nor the "inner" operator $\mathcal{B}_{0, \theta}^{\text {hom }}$ is suitable for order $O(\varepsilon)$ estimates. This leads to an interpretation of $\mathcal{B}_{\varepsilon, \theta}^{\text {hom }}$ as a non-trivial matching of $\mathcal{B}_{0}$ and $\mathcal{B}_{0, \theta}^{\text {hom }}$ in the boundary layer necessary to achieve order $O(\varepsilon)$ estimates. This interpretation is further supported by the following result, which states that by extending $\mathcal{B}_{0}$ and $\mathcal{B}_{0, \theta}^{\text {hom }}$ into the boundary layer one can only achieve $O\left(\varepsilon^{2 / 3}\right)$-estimates.

Corollary 8.2. For all $\varepsilon>0, \alpha \in(0,1)$, denote $B_{\varepsilon^{\alpha-1}}(0):=\left\{\theta:|\theta|<\varepsilon^{\alpha-1}\right\}$ and consider the operators

$$
\begin{aligned}
\mathcal{S}^{\varepsilon, \alpha}:= & \mathcal{U}_{\varepsilon}^{-1}\left(\int_{\theta \in B_{\varepsilon^{\alpha-1}}(0)}^{\oplus} \mathcal{I}\left(\mathcal{B}_{0, \theta}^{\text {hom }}+I\right)^{-1} \mathcal{I}^{-1} \mathcal{P}_{\mathrm{f}} \mathrm{d} \theta\right. \\
& \left.+\int_{\theta \in \varepsilon^{-1} Q^{\prime} \backslash B_{\varepsilon^{\alpha-1}}(0)}^{\oplus}\left[\mathrm{e}^{-\mathrm{i} \varepsilon \theta \cdot}\right]\left(\mathcal{B}_{0}+I\right)^{-1} \mathcal{P}_{0}\left[\mathrm{e}^{\mathrm{i} \varepsilon \theta \cdot}\right] \mathrm{d} \theta\right) \mathcal{U}_{\varepsilon}
\end{aligned}
$$

There exists a constant $C=C(\alpha)$ independent of $\varepsilon$ such that

$$
\left\|\left(\mathcal{A}^{\varepsilon}+I\right)^{-1}-\mathcal{S}^{\varepsilon, \alpha}\right\|_{L^{2}\left(\mathbb{R}^{d}\right) \rightarrow L^{2}\left(\mathbb{R}^{d}\right)} \leq C\left(\varepsilon^{\alpha}+\varepsilon^{2(1-\alpha)}\right) .
$$

In particular, minimising the estimates over $\alpha$, the operators $\mathcal{S}^{\varepsilon, \alpha}$ are shown to be $\varepsilon^{2 / 3}$-close in the operator norm to the resolvents $\left(\mathcal{A}^{\varepsilon}+I\right)^{-1}$.

The above result can be extended to get error estimates in terms of higher powers of $\varepsilon$, by including further correcting terms from the asymptotic expansions for the functions $u_{\theta}^{\varepsilon}$ (Section 5) in the new "inner" region $|\theta| \leq \varepsilon^{\beta-1}$ and for the functions $w_{\varepsilon, \varkappa}$ (the present section above) in the new "outer" region $|\varkappa| \geq \varepsilon^{1-\gamma}$ (equivalently, $|\theta| \geq \varepsilon^{-\gamma}$ ) for $\beta, \gamma>0$ such that $\beta+\gamma \leq 1$. A direct construction of this kind yields operator-norm estimates for $\left(\mathcal{A}^{\varepsilon}+I\right)^{-1}$ of order $O\left(\varepsilon^{\beta(N+1)}+\varepsilon^{2 \gamma(N+1)}\right)$, which is optimised to $O\left(\varepsilon^{2(N+1) / 3}\right)$ by setting $\beta=2 / 3$, $\gamma=1 / 3$. Further, using the fact that the two expansions coincide in the "overlapping" region $\varepsilon^{-\gamma} \leq|\theta| \leq \varepsilon^{\beta-1}$, when $\beta+\gamma<1$, one can then construct 
a $\theta$-uniform asymptotic expansion, whose $N$-th truncation [that is replacing " $\infty$ " with " $N$ " in (5.1) and (8.2)] yields an error of order $O\left(\varepsilon^{\sigma(N+1)}\right)$ with $0<\sigma \leq 1$. This asymptotic procedure is well known in the analysis of parameter-dependent functions of the spatial variables $(x, y)$, see for example [7], especially in the context spatial representation of solutions to PDE. Our approach exploits similar ideas in the "dual" formulation, with respect to the quasimomenta $(\theta, \varkappa)$, which in our view has a potential to yield powerful results for operator-norm resolvent estimates for a general class of parameter-dependent families of operators. In particular, the proof of our main result (Theorem 4.1) can be viewed as a matching procedure that achieves $\sigma=1$ for the case $N=0$.

\section{Spectra of the Operators $\mathcal{B}_{\varepsilon, \theta}^{\text {hom }}$}

Using the definition of the form $\mathfrak{b}_{\varepsilon, \theta}^{\text {hom }}$, see Section 4, we infer that a pair $(c, u) \in$ $\mathcal{H}_{0}$ is an eigenvector of the operator $\mathcal{B}_{\varepsilon, \theta}^{\text {hom }}$ corresponding to an eigenvalue $\lambda$ if and only if

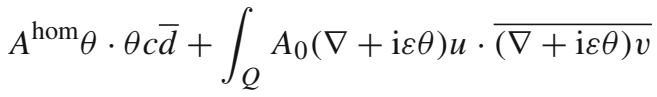

$$
\begin{aligned}
& =\lambda \int_{Q}(c+u) \overline{(d+v)} \quad \forall(d, v) \in \mathcal{H}_{0} .
\end{aligned}
$$

Setting $v=0$ in (9.1) with an arbitrary $d \in \mathbb{C}$ yields

$$
A^{\mathrm{hom}} \theta \cdot \theta c=\lambda\left(c+\int_{Q} u\right) .
$$

Further, setting $d=0$ in (9.1) with an arbitrary $v \in H_{0}^{1}\left(Q_{0}\right)$ yields

$$
\int_{Q} A_{0}(\nabla+\mathrm{i} \varepsilon \theta) u \cdot \overline{(\nabla+\mathrm{i} \varepsilon \theta) v}=\lambda \int_{Q}(c+u) \bar{v},
$$

from which we deduce that either $\lambda \in S_{0}:=\left\{\lambda_{j}\right\}_{j=0}^{\infty}$, the set of eigenvalues of the operator $\mathcal{A}_{0}=-\nabla \cdot A_{0} \nabla$ in $L^{2}(Q)$, defined by the sesquilinear form

$$
\mathfrak{a}_{0}(u, v):=\int_{Q} A_{0} \nabla u \cdot \overline{\nabla v}, \quad u, v \in H_{0}^{1}\left(Q_{0}\right),
$$

on the maximal possible domain $D\left(\mathcal{A}_{0}\right)$, or $\lambda \notin S_{0}$ and

$$
u=\lambda c \sum_{j=0}^{\infty}\left(\lambda_{j}-\lambda\right)^{-1}\left(\int_{Q_{0}} \varphi_{j}^{*}\right) \overline{\varphi_{j}^{*}},
$$

where $\varphi_{j}^{*}(y):=\varphi_{j}(y) \exp (\mathrm{i} \varepsilon \theta \cdot y), y \in Q$, and $\varphi_{j}$ is the eigenfunction of $\mathcal{A}_{0}$ corresponding to the eigenvalue $\lambda_{j}, j=0,1, \ldots$ (We assume that the eigenvalues are ordered by magnitude, $\lambda_{0}<\lambda_{1} \leq \lambda_{2} \leq \ldots$, where multiple eigenvalues are appear the number of times equal to their multiplicity and that $\varphi_{j}, j=0,1,2, \ldots$, 
are real-valued and linearly independent.) In the former case one has $c=0$ and (9.2) implies $\int_{Q} u=0$, while in the latter case $c \in \mathbb{C}$ is arbitrary and by substituting (9.3) into (9.2) one gets

$$
A^{\operatorname{hom}} \theta \cdot \theta=\lambda\left(1+\lambda \sum_{j=0}^{\infty}\left(\lambda_{j}-\lambda\right)^{-1}\left|\int_{Q_{0}} \varphi_{j}^{*}\right|^{2}\right) .
$$

The expression

$$
\beta(\lambda):=\lambda\left(1+\lambda \sum_{j=0}^{\infty}\left(\lambda_{j}-\lambda\right)^{-1}\left(\int_{Q_{0}} \varphi_{j}\right)^{2}\right),
$$

obtained by setting $\varepsilon \theta=0$ in the right-hand side of (9.4), appeared in the work [14], where the behaviour of the spectra of the operators $\mathcal{A}^{\varepsilon}$ was analysed. In particular, our main theorem above (Theorem 4.1) implies the result of [15] on convergence of the spectra of $\mathcal{A}^{\varepsilon}$, as follows.

Theorem 9.1. The spectra of the operators $\mathcal{A}^{\varepsilon}$ converge in the Hausdorff sense as $\varepsilon \rightarrow 0$ to the union of the set $S_{0}$ and the set

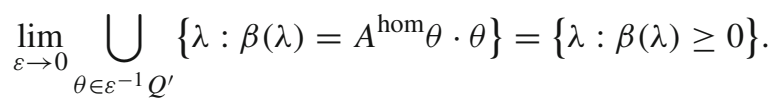

\section{Two Particular Examples of the Family $A^{\varepsilon}$}

Here we discuss two model cases included in our analysis that have emerged in the literature.

\subsection{Classical Homogenisation: $Q_{0}=\varnothing$}

This is the case when $V$ consists of constant functions on $Q$. The inequality (6.3) trivially holds for $\varkappa \neq 0$, and for $\varkappa=0$ it takes the form of the usual Poincare inequality for functions with zero mean over $Q$. Clearly, the space $\mathcal{H}_{0}$ is isometric to $\mathbb{C}$ and the operator family $\mathcal{R}_{\varepsilon}$ consists of just one element, the resolvent of the usual homogenised operator

$$
\mathcal{A}^{\text {hom }} v:=-\nabla \cdot A^{\text {hom }} \nabla,
$$

where the matrix $A^{\text {hom }}$ is given by (4.2). Indeed, in this example the operator family $\mathcal{B}_{\varepsilon, \theta}^{\text {hom }}$ does not depend on $\varepsilon$ and for each specific value of $\varepsilon$ represents $\theta$-components of the direct fibre decomposition of the operator $\mathcal{A}^{\text {hom }}$ treated as an operator with $\varepsilon$-periodic coefficients, that is

$$
\mathcal{U}_{\varepsilon}^{-1} \mathcal{A}^{\text {hom }} \mathcal{U}_{\varepsilon}=\int_{\varepsilon^{-1} Q^{\prime}}^{\oplus} \theta \cdot A^{\text {hom }} \theta \mathrm{d} \theta=\int_{\varepsilon^{-1} Q^{\prime}}^{\oplus} \mathcal{I}_{\varepsilon, \theta}^{\text {hom }} \mathcal{I}^{-1} \mathrm{~d} \theta .
$$

Hence in this case Theorem 4.1 recovers the result of Birman and Suslina [3] regarding the resolvent convergence estimates for classical homogenisation in $\mathbb{R}^{d}$. 


\subsection{The "Double Porosity" Problem: $Q_{0} \neq \emptyset,\left.A_{0}\right|_{Q_{1}}=0$}

This was considered in the work by ZHIKOv [15], where the spectrum of doubleporosity problems in $\mathbb{R}^{d}$ was analysed, following an earlier work [14] concerning double-porosity models in bounded domains.

The paper [15] contains a proof of the strong two-scale convergence of the sequence of solutions $u=u^{\varepsilon}$ to the problems (2.1) to the solution $\left(v_{1}, v_{0}\right) \in$ $\mathcal{H}^{\mathrm{dp}}:=H^{1}\left(\mathbb{R}^{d}\right) \times L^{2}\left(\mathbb{R}^{d}, H_{0}^{1}\left(Q_{0}\right)\right), v_{0}=v_{0}(x, y)$, of the problem

$$
\mathfrak{a}^{\mathrm{dp}}\left(\left(v_{1}, v_{0}\right),\left(\varphi_{1}, \varphi_{0}\right)\right)+\int_{\mathbb{R}^{d}} \int_{Q}\left(v_{1}+v_{0}\right) \overline{\left(\varphi_{1}+\varphi_{0}\right)}=\int_{\mathbb{R}^{d}} \int_{Q} f \overline{\left(\varphi_{1}+\varphi_{0}\right)},
$$

where the form $\mathfrak{a}^{\mathrm{dp}}$, with $D\left(\mathfrak{a}^{\mathrm{dp}}\right)=\mathcal{H}^{\mathrm{dp}}$, is given by

$$
\mathfrak{a}^{\mathrm{dp}}\left(\left(v_{1}, v_{0}\right),\left(\varphi_{1}, \varphi_{0}\right)\right):=\int_{\mathbb{R}^{d}} A^{\mathrm{hom}} \nabla v_{1} \cdot \overline{\nabla \varphi_{1}}+\int_{\mathbb{R}^{d}} \int_{Q} A_{0} \nabla_{y} v_{0} \cdot \overline{\nabla_{y} \varphi_{0}} .
$$

The author of [15] refers to the operator $\mathcal{A}^{\mathrm{dp}}$ generated by $\mathfrak{a}^{\mathrm{db}}$ as the homogenised operator for the family $\mathcal{A}^{\varepsilon}$ and proves that the spectra of $\mathcal{A}^{\varepsilon}$ converge to the spectrum of $\mathcal{A}^{\mathrm{dp}}$ as $\varepsilon \rightarrow 0$. For continuous right-hand sides $f$, the strong two-scale convergence result of [14] implies that

$$
\left\|u^{\varepsilon}-v_{1}(x)-\widetilde{v}_{0}\left(x, \frac{x}{\varepsilon}\right)\right\|_{L^{2}\left(\mathbb{R}^{d}\right)}<C \varepsilon,
$$

where $\widetilde{v}_{0}$ is the $Q$-periodic extension of the function $v_{0}=v_{0}(x, y)$ after setting it to zero for $y \in Q_{1}$. In the estimate (10.1) the constant $C=C(f)>0$ is independent of $\varepsilon$, but it can not be replaced by $C\|f\|_{L^{2}\left(\mathbb{R}^{d}\right)}$ with a constant $C$ that is independent of both $\varepsilon$ and $f$. (In other words, there are sequences $f^{\varepsilon}$ that are bounded in $L^{2}\left(\mathbb{R}^{d}\right)$ and are such that $C\left(f^{\varepsilon}\right) \rightarrow \infty$ as $\varepsilon \rightarrow 0$.)

The estimate (10.1) can also be written in the form

$$
\left\|\left(\mathcal{A}^{\varepsilon}+I\right)^{-1} f-\mathcal{S}^{\varepsilon}\left(\mathcal{A}^{\mathrm{dp}}+I\right)^{-1} f\right\|_{L^{2}\left(\mathbb{R}^{d}\right)}<C(f) \varepsilon,
$$

where in the expression $\left(\mathcal{A}^{\mathrm{dp}}+I\right)^{-1} f$ the function $f$ is treated as an element of $L^{2}\left(\mathbb{R}^{d} \times Q\right)$, and the operator $\mathcal{S}^{\varepsilon}: L^{2}\left(\mathbb{R}^{d} \times Q\right) \rightarrow L^{2}\left(\mathbb{R}^{d}\right)$ is defined by $\left(\mathcal{S}^{\varepsilon} u\right)(x)=u(x, x / \varepsilon), x \in \mathbb{R}^{d}$. The inequality (10.2), however, can not be upgraded to an operator-norm resolvent type statement, in view of the fact that the difference of the corresponding spectral projections on a neighbourhood of any point of the form $\left(\lambda_{\infty}+I\right)^{-1}$, where $\lambda_{\infty}$ is such that $\beta(\lambda) \rightarrow \infty$ as $\lambda \rightarrow \lambda_{\infty}$, does not go to zero in the operator norm as $\varepsilon \rightarrow 0$. (Such points $\lambda_{\infty}$ are the eigenvalues of the operator $\mathcal{A}_{0}$ that have at least one eigenfunction with non-zero integral over $Q$.) Our estimate (4.3) therefore rectifies this drawback and captures the operator-norm resolvent asymptotic behaviour of the sequence $\mathcal{A}^{\varepsilon}$.

Acknowledgments. This work was carried out under the financial support of the Leverhulme Trust (Grant RPG-167 "Dissipative and non-self-adjoint problems") and the Engineering and Physical Sciences Research Council (Grant EP/L018802/1 "Mathematical foundations of metamaterials: homogenisation, dissipation and operator theory"). We are also grateful to the anonymous reviewer for their insightful comments. 
Open Access This article is distributed under the terms of the Creative Commons Attribution 4.0 International License (http://creativecommons.org/licenses/by/4.0/), which permits unrestricted use, distribution, and reproduction in any medium, provided you give appropriate credit to the original author(s) and the source, provide a link to the Creative Commons license, and indicate if changes were made.

\section{References}

1. Allaire, G.: Homogenization and two-scale convergence. SIAM J. Math. Anal. 23, 1482-1518 (1992)

2. Bensoussan, A., Lions, J.-L., Papanicolaou, G. C.: Asymptotic Analysis for Periodic Structures. North-Holland, Amsterdam, 1978

3. Birman, M.Sh., Suslina, T.A.: Second order periodic differential operators. Threshold properties and homogenisation. St. Petersb. Math. J. 15 (5), 639-714 (2004)

4. Conca, C., Vanninathan, M.: Homogenisation of periodic structures via Bloch decomposition. SIAM J. Appl. Math. 57, 1639-1659 (1997)

5. Griso, G.: Interior error estimate for periodic homogenisation. Anal. Appl. 4(1), 61-79 (2006)

6. Hempel, R., Lienau, K.: Spectral properties of periodic media in the large coupling limit. Commun. Partial Differ. Equ. 25, 1445-1470 (2000)

7. IL'IN, A.M.: Matching of Asymptotic Expansions of Solutions of Boundary Value Problems. American Mathematical Society, Providence, 1992

8. JiKov, V.V., Kozlov, S.M., Oleinik, O.A.: Homogenization of Differential Operators and Integral Functionals. Springer, Berlin, 1994

9. Kamotski, I.V., Smyshlyaev, V.P.: Two-scale homogenization for a class of partially degenerating PDE systems. arXiv:1309.4579 (2011) (arXiv preprint)

10. Kenig, C.E., Lin, F., Shen, Z.: Convergence rates in $L^{2}$ for elliptic homogenization problems. Arch. Ration. Mech. Anal. 203(3), 1009-1036 (2012)

11. Landau, L.D., Lifshitz, E.M.: Electrodynamics of Continuous Media. Pergamon Press, Oxford, 1960

12. Suslina, T. A.: Homogenization of the Dirichlet problem for elliptic systems: L2operator error estimates. Mathematika 59, 463-476 (2013)

13. Zнікоv, V. V.: Spectral approach to asymptotic problems in diffusion. Differ. Equ. 25, 33-39 (1989)

14. Zhiкоv, V.V.: On an extension of the method of two-scale convergence and its applications. Sb. Math. 191(7), 973-1014 (2000)

15. Zнiкоv, V.V.: On gaps in the spectrum of some divergence elliptic operators with periodic coefficients. St. Petersb. Math. J. 16 (5), 773-719 (2005)

16. Zhikov, V.V., Pastukhova, S.E.: On operator estimates for some problems in homogenization theory. Russ. J. Math. Phys. 12 (4), 515-524 (2005)

Department of Mathematical Sciences,

University of Bath,

Claverton Down,

Bath BA27AY, UK.

e-mail: K.Cherednichenko@bath.ac.uk

(Received June 24, 2014 / Accepted July 24, 2015)

Published online September 7, 2015 - (C) The Author(s) (2015)

This article is published with open access at Springerlink.com 